\title{
The OLYMPUS Experiment
}

\author{
The OLYMPUS Collaboration
}

R. Milner ${ }^{\mathrm{a}}$, D.K. Hasella,*, M. Kohl ${ }^{\mathrm{b}}$, U. Schneekloth ${ }^{\mathrm{c}}$, N. Akopov ${ }^{\mathrm{d}}$, R. Alarcon ${ }^{\mathrm{e}}$, V.A. Andreev ${ }^{\mathrm{f}}$, O. Ates ${ }^{\mathrm{b}}$, A. Avetisyan ${ }^{\mathrm{d}}$, D. Bayadilov ${ }^{\mathrm{g}}$, R. Beck $^{\mathrm{g}}$, S. Belostotski $^{\mathrm{f}}$, J.C. Bernauer ${ }^{\mathrm{a}}$, J. Bessuille ${ }^{\mathrm{a}}$, F. Brinker ${ }^{\mathrm{c}}$, B. Buck ${ }^{\mathrm{a}}$, J.R. Calarco ${ }^{\mathrm{h}}$, V. Carassiti ${ }^{\mathrm{i}}$, E. Cisbani ${ }^{\mathrm{j}}$, G. Ciullo ${ }^{\mathrm{i}}$, M. Contalbrigo ${ }^{\mathrm{i}}$, N. D’Ascenzo $^{\mathrm{c}}$, R. De Leo $^{\mathrm{k}}$, J. Diefenbach ${ }^{\mathrm{b}, 1}$, T.W. Donnelly ${ }^{\mathrm{a}}$, K. Dow ${ }^{\mathrm{a}}$, G. Elbakian ${ }^{\mathrm{d}}$, D. Eversheim ${ }^{\mathrm{g}}$, S. Frullani ${ }^{\mathrm{j}}$, Ch. Funke E $^{\mathrm{g}}$, G. Gavrilov ${ }^{\mathrm{f}}$, B. Gläser ${ }^{\mathrm{l}}$, N. Görrissen ${ }^{\mathrm{c}}$, J. Hauschildt ${ }^{\mathrm{c}}$, B.S. Henderson ${ }^{\mathrm{a}}$, Ph. Hoffmeister ${ }^{\mathrm{g}}$, Y. Holler ${ }^{\mathrm{c}}$, L.D. Ice ${ }^{\mathrm{e}}$, A. Izotov ${ }^{\mathrm{f}}$, R. Kaiser ${ }^{\mathrm{m}}$, G. Karyan ${ }^{\mathrm{d}}$, J. Kelsey ${ }^{\mathrm{a}}$, D. Khaneft ${ }^{l}$, P. Klassen ${ }^{\mathrm{g}}$, A. Kiselev ${ }^{\mathrm{f}, 2}$, A. Krivshich ${ }^{\mathrm{f}}$, I. Lehmann ${ }^{\mathrm{m}, 3}$, P. Lenisa ${ }^{\mathrm{i}}$, D. Lenz ${ }^{\mathrm{c}}$, S. Lumsden ${ }^{\mathrm{m}}, \mathrm{Y} . \mathrm{Ma}^{\mathrm{l}, 4}, \mathrm{~F}$. Maas $^{\mathrm{l}}, \mathrm{H}$. Marukyan $^{\mathrm{d}}$, O. Miklukho ${ }^{\mathrm{f}}$, A. Movsisyan ${ }^{\mathrm{i}, \mathrm{d}}$, M. Murray ${ }^{\mathrm{m}}$, Y. Naryshkin ${ }^{\mathrm{f}}$, C. O'Connor ${ }^{\mathrm{a}}$, R. Perez Benito ${ }^{\mathrm{l}}$, R. Perrino ${ }^{\mathrm{k}}$, R.P. Redwine ${ }^{\mathrm{a}}$, D. Rodríguez Piñeiro ${ }^{\mathrm{l}}$, G. Rosner ${ }^{\mathrm{m}, 3}$, R.L. Russell ${ }^{\mathrm{a}}$,

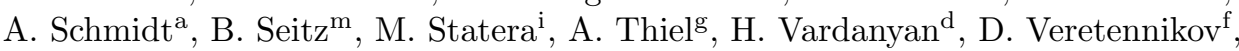

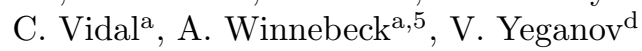

\author{
${ }^{a}$ Massachusetts Institute of Technology, Cambridge, MA, USA \\ ${ }^{b}$ Hampton University, Hampton, VA, USA \\ ${ }^{c}$ Deutsches Elektronen-Synchrotron DESY, Hamburg, Germany \\ ${ }^{d}$ Alikhanyan National Science Laboratory (Yerevan Physics Institute), Yerevan, Armenia \\ ${ }^{e}$ Arizona State University, Tempe, AZ, USA

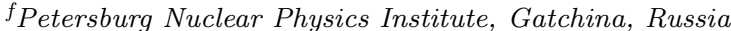 \\ ${ }^{g}$ Friedrich Wilhelms Universität, Bonn, Germany \\ ${ }^{h}$ University of New Hampshire, Durham, NH, USA \\ ${ }^{i}$ Università di Ferrara and Istituto Nazionale di Fisica Nucleare, Ferrara, Italy \\ ${ }^{j}$ Istituto Superiore di Sanità and Istituto Nazionale di Fisica Nucleare, Rome, Italy \\ ${ }^{k}$ Istituto Nazionale di Fisica Nucleare, Bari, Italy \\ ${ }^{l}$ Johannes Gutenberg-Universität, Mainz, Germany \\ ${ }^{m}$ University of Glasgow, Glasgow, United Kingdom
}

\begin{abstract}
The OLYMPUS experiment was designed to measure the ratio between the positronproton and electron-proton elastic scattering cross sections, with the goal of determining the contribution of two-photon exchange to the elastic cross section. Two-photon exchange might resolve the discrepancy between measurements of the proton form factor ratio, $\mu_{p} G_{E}^{p} / G_{M}^{p}$, made using polarization techniques and those made in unpolarized experiments. OLYMPUS operated on the DORIS storage ring at DESY, alternating between $2.01 \mathrm{GeV}$ electron and positron beams incident on an internal hydrogen gas target. The experiment used a toroidal magnetic spectrometer instrumented with drift chambers and time-of-flight detectors to measure rates for elastic scattering over the polar angular range of approximately $25^{\circ}-75^{\circ}$. Symmetric Møller/Bhabha calorimeters at $1.29^{\circ}$ and telescopes of GEM and MWPC detectors at $12^{\circ}$ served as luminosity monitors. A total luminosity of approximately $4.5 \mathrm{fb}^{-1}$ was collected over two running periods in 2012 .
\end{abstract}


This paper provides details on the accelerator, target, detectors, and operation of the experiment.

Keywords: elastic electron scattering, elastic positron scattering, two-photon exchange, form-factor ratio

2010 MSC: 25.30.Bf, 25.30.Hm, 13.60.Fz, 13.40.Gp, 29.30.-h

\section{Introduction}

Electron scattering has long been an important tool for studying the structure of nucleons. The strength of the technique lies in the predominantly electromagnetic nature of the interaction. The electron is, to the best of our knowledge, a point-particle, and its interaction is well described by quantum electrodynamics. The interaction is mediated by a virtual photon, whose momentum transfer sets a size scale for the structures that are probed in the scattering reaction. A low-momentum virtual photon can only "see" the size of the nucleon, but by increasing the momentum transfer, the photon is sensitive to the nucleon's internal distribution of charge and magnetism, parameterized by form factors $G_{E}$ and $G_{M}$. At even higher momentum transfers, deep inelastic scattering reveals the distributions of the quarks and gluons, which are ultimately responsible for the observed form factors. The synthesis of data at all different momentum scales can verify and guide our theoretical understanding of the nucleon.

Polarized beams and targets offer another window into the structure of nucleons. Recently, measurements of the electric to magnetic form factor ratio of the proton, $\mu_{p} G_{E}^{p} / G_{M}^{p}$, using polarization techniques [1-13] have shown a dramatic discrepancy at high four-momentum transfer, $Q^{2}$, in comparison with the ratio obtained using the traditional Rosenbluth technique in unpolarized cross section measurements [14 19, highlighted in Fig. 1 by a selection of data sets. This discrepancy might arise from a significant contribution to the elastic electron-proton cross section from hard two-photon exchange [21 26], a process that is neglected in the standard radiative corrections procedures. Since there is no theoretical consensus on the size of this contribution [21 31, definitive measurements are needed to determine if two-photon exchange resolves the form factor discrepancy.

To address this question, the OLYMPUS experiment was proposed to measure the ratio between the positron-proton and electron-proton elastic scattering cross sections. In the single-photon exchange approximation this ratio is unity. At next-to-leading order, the interference of the one-photon and two-photon exchange diagrams changes sign between electron and positron scattering. The two photon exchange effect is expected to depend on the lepton scattering angle, $\theta$, or virtual photon polarization,

\footnotetext{
* Corresponding Author

Email address: hasell@mit.edu (D.K. Hasell)

${ }^{1}$ Currently with Johannes Gutenberg-Universität, Mainz, Germany

${ }^{2}$ Currently with Brookhaven National Laboratory, Upton, NY, USA

${ }^{3}$ Also with the Facility for Antiproton and Ion Research, Darmstadt, Germany

${ }^{4}$ Currently with RIKEN, Nishina Center, Advanced Meson Science Laboratory, Japan

${ }^{5}$ Currently with Varian Medical Systems, Bergisch Gladbach, Germany 


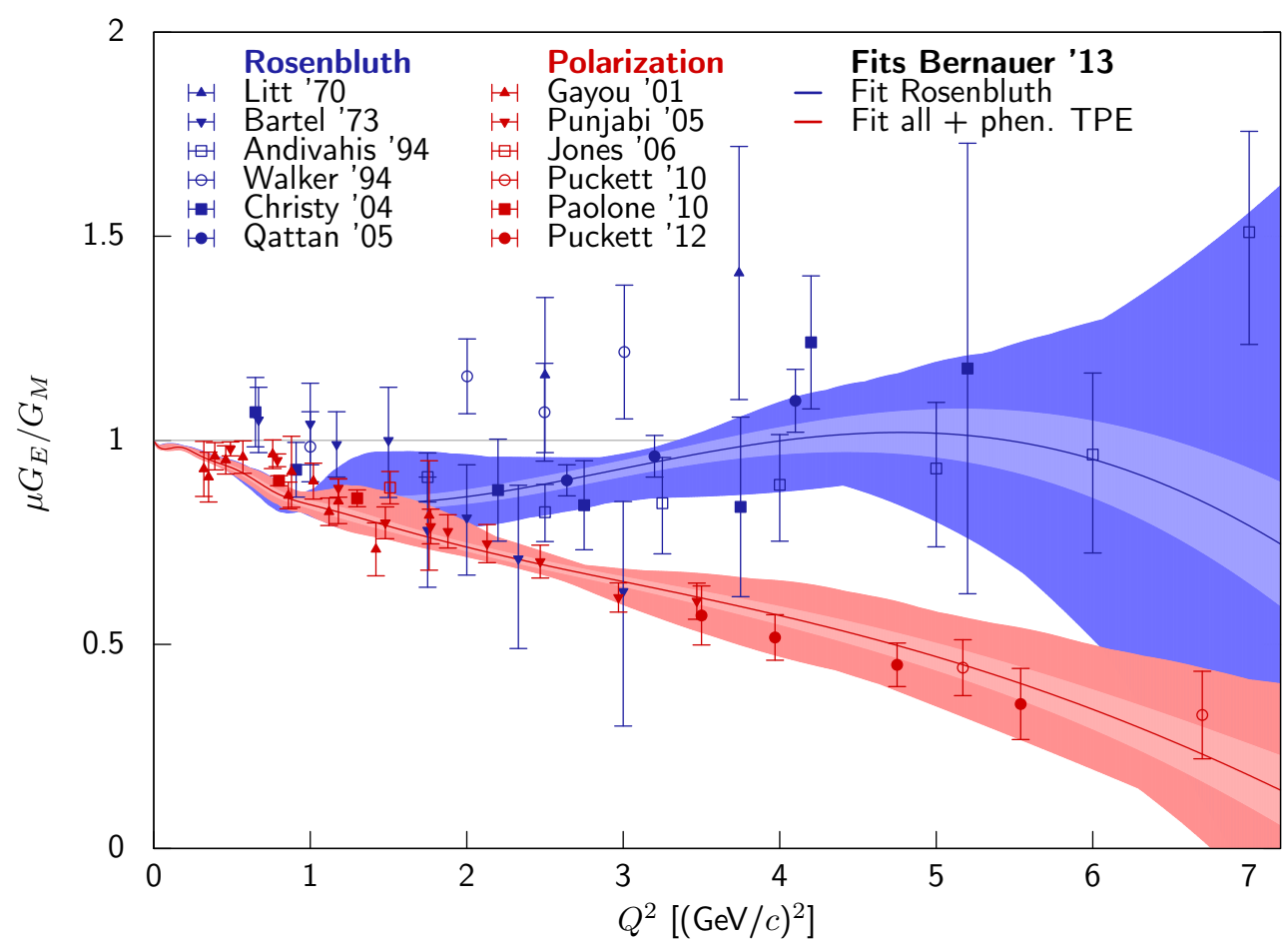

Fig. 1: The ratio of proton form factors, $\mu_{p} G_{E}^{p} / G_{M}^{p}$, as a function of $Q^{2}$ from (unpolarized) Rosenbluth measurements [14 19] are inconsistent with recent data collected using polarization techniques [8 13]. Also shown are the ratios from fits [20] of the form factors to the world dataset. The light shaded bands show statistical uncertainty and the dark shaded bands show model uncertainty added linearly. 
$\epsilon=\left[1+2\left(1+\frac{Q^{2}}{4 M_{2}^{2}}\right) \tan ^{2}(\theta / 2)\right]^{-1}$, where $M_{p}$ is the proton mass. Measurements from the 1960s indicated some deviation in the ratio from unity, but the uncertainties were large, as can be seen in Fig. 2. OLYMPUS seeks to measure the ratio with uncertainty of less than $1 \%$ over the range $0.4(\mathrm{GeV} / \mathrm{c})^{2} \leq Q^{2} \leq 2.2(\mathrm{GeV} / \mathrm{c})^{2}$ for a single beam energy $E=2.01 \mathrm{GeV}$.

OLYMPUS was approved for three months of dedicated operation at the DORIS electron/positron storage ring at DESY, in Hamburg, Germany. Beams of electrons or positrons were directed on an internal hydrogen gas target, with the scattered leptons and recoiling protons detected in coincidence over a wide range of scattering angles $\left(25^{\circ} \leq \theta \leq 75^{\circ},-15^{\circ} \leq \phi \leq 15^{\circ}\right)$. The target was designed and built at MIT and installed on the DORIS ring. The former BLAST detector was shipped from MITBates to DESY and placed around the target. The detector used a toroidal magnetic field with a left/right symmetric arrangement of tracking detectors and time of flight scintillators. In addition, three new detector systems were designed and built to monitor the luminosity during the experiment. Telescopes mounted at $\theta=12^{\circ}$ consisted of triple GEM detectors from Hampton University with readout electronics from INFN Rome and MWPC detectors from PNPI. Symmetric Møller/Bhabha calorimeters from Mainz were positioned at $1.29^{\circ}$. The Bonn group provided the software and hardware for the data acquisition system. The trigger and slow control systems were developed by MIT.

The OLYMPUS experiment collected data in two periods: the February period (January 20 - February 27, 2012) and the fall period (October 24, 2012 - January 2, 2013). During the February period, the beam species was typically changed daily, and the magnet polarity was changed randomly, but equally, every 6 hours. For the February data run, there was a leak in the target gas supply that caused only a fraction of the measured flow to reach the target cell. Because of this, a lower than expected luminosity was obtained. The gas leak was repaired in the summer so that it was possible to achieve high luminosity in the fall period. However, it was discovered that at high luminosity and negative magnet polarity too many electrons were bent into the drift chambers, preventing their operation. After several tests and attempts to remedy this, it was decided to operate at high luminosity but primarily with positive magnet polarity for most of the fall period.

The following sections describe the accelerator, target, detectors, data acquisition, and operation in more detail.

\section{DORIS Storage Ring at DESY}

The DORIS storage ring at DESY originally began operation in 1974 as an electronelectron and electron-positron collider. After its long and successful operation for particle physics research, DORIS was dedicated to synchrotron radiation studies in 1993. Since DORIS had access to both positron and electron sources and could circulate both species at several $\mathrm{GeV}$ energies, it met the requirements for the OLYMPUS experiment. Additionally, the infrastructure at the location in the beamline of the former ARGUS experiment 38. provided an excellent match to the size and needs of OLYMPUS. In 2009, the shutdown of DORIS was scheduled for the end of 2012, placing a tight time constraint on OLYMPUS. 


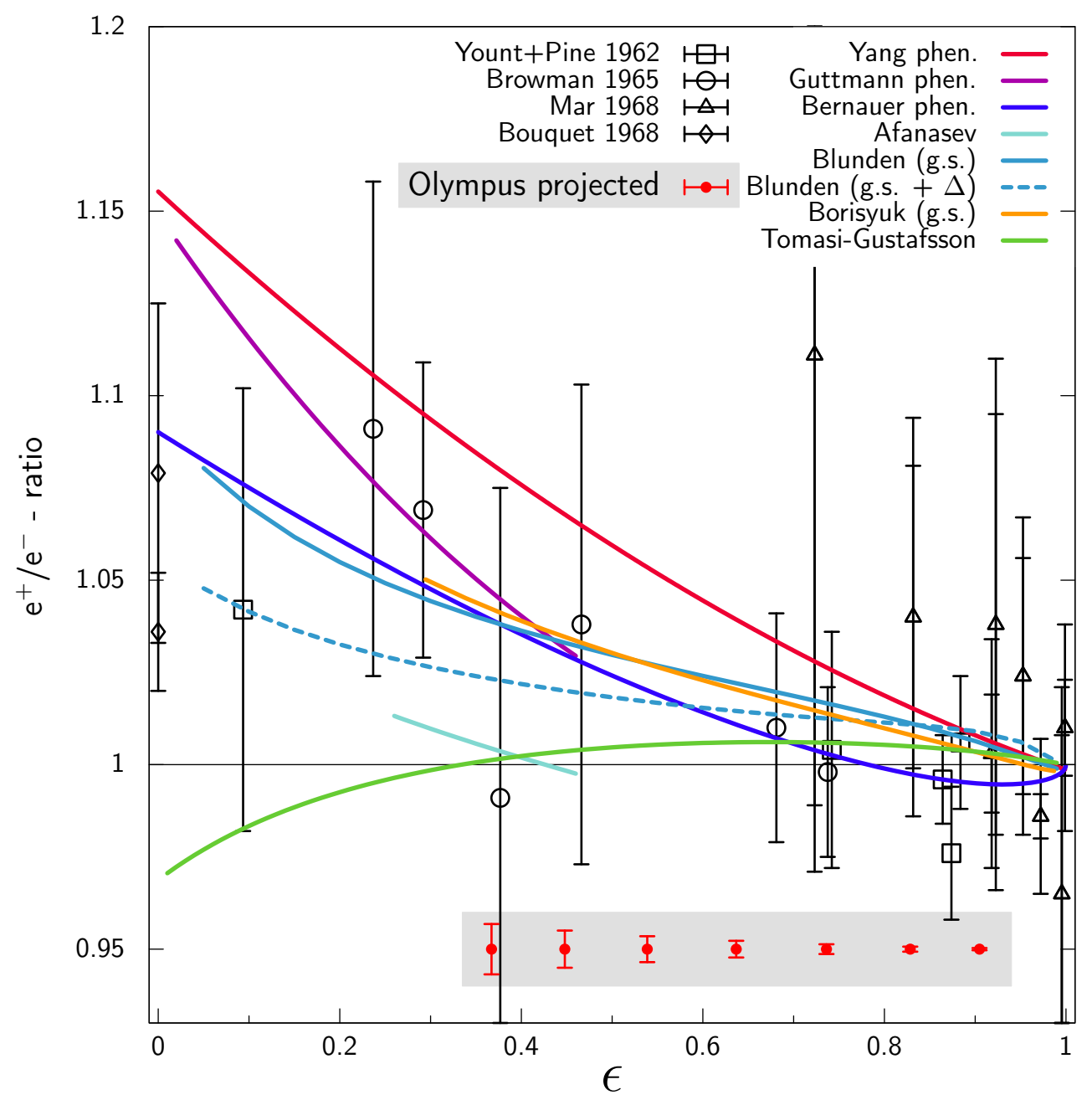

Fig. 2: The ratio of positron to electron elastic scattering cross sections at a beam energy of $2 \mathrm{GeV}$ as a function of $\epsilon$ showing phenomenological predictions [20, 32, 33], a selection of theoretical calculations of hard two-photon exchange [22 28], and the projected OLYMPUS uncertainties. Also shown are existing ratio data 34 37 that were not taken at $2 \mathrm{GeV}$, plotted at their corresponding values of $\epsilon$. 
Although the DORIS accelerator and the ARGUS detector site were well suited to the OLYMPUS experiment, several modifications were required. In particular, a number of considerations were necessary to allow DORIS to continue to operate as a synchrotron light source after OLYMPUS was installed (although not during OLYMPUS data taking). These included:

- RF cavities that had been installed at the detector site had to be relocated $26 \mathrm{~m}$ upstream.

- An additional quadrupole was installed on each side $( \pm 7 \mathrm{~m})$ of the OLYMPUS interaction region to reduce the beam size for the OLYMPUS target while not significantly affecting the beam profile in synchrotron radiation source elements. This was necessary due to the impracticality of removing the OLYMPUS target for synchrotron runs.

- The OLYMPUS target required cooling during synchrotron radiation runs due to the wakefield heating caused by the $150 \mathrm{~mA}, 4.5 \mathrm{GeV}, 5$-bunch beam.

- A number of tests and improvements were required to achieve the 10-bunch, 2.01 $\mathrm{GeV}$ beam conditions for OLYMPUS operation with adequate currents and lifetimes, including the implementation of a multi-bunch feedback system.

A key feature of the OLYMPUS experiment was the frequent switching between electron and positron beams. The DORIS pre-accelerators were already able to switch between electrons and positrons within approximately 10 minutes, but the extraction from the pre-accelerators to DORIS, the transport line, and the DORIS ring needed several modifications:

- The high voltage pulse power supplies for the pre-accelerator extraction and the DORIS injection kickers had to be rebuilt.

- The septa magnets for pre-accelerator extraction and DORIS injection were modified to serve as bipolar devices.

- Remotely-controlled polarity switches for a number of 800 A magnet power supplies had to be constructed and installed.

The daily switching of the beam species for OLYMPUS posed a challenge during the fall period when DORIS and the PETRA storage ring operated in parallel. The two rings shared the same pre-accelerators, and PETRA only circulated positrons. The procedure for switching the polarity of the pre-accelerators was optimized so that PETRA could be refilled with positrons in approximately five minutes, causing only a small delay in electron refills for DORIS.

Since the injection into DORIS occurred at full energy, it was possible to run in top-up mode. This allowed OLYMPUS to operate with a higher target density while maintaining a high average beam current, while also keeping the beam current at a more constant level. The injection process was optimized in order to minimize beam losses, which prevented excessive rates and high voltage trips in the OLYMPUS detectors.

The radiation levels in the region downstream of the experiment increased when gas was added to the target, and additional shielding was installed to account for this. Also, 
the beam scrapers upstream of the experiment were optimized to minimize the noise rates in the experiment.

To monitor the beam energy, a dipole reference magnet was installed in series with the DORIS dipole magnets. This magnet included a rotating coil to measure the integrated field strength. The accelerator archive system monitored all relevant data, power supply currents for all magnets, beam position data, scraper positions, etc. and provided much of this information to the OLYMPUS slow control system.

\section{Target and Vacuum Systems}

The OLYMPUS experiment used an unpolarized, internal hydrogen gas target cooled to below $70 \mathrm{~K}$. The hydrogen gas flowed into an open-ended, $600 \mathrm{~mm}$ long, elliptical target cell (Sec. 3.1). The target cell was housed in a scattering chamber (Sec. 3.2) that had thin windows between the cell and the detectors. The target system was designed to withstand both OLYMPUS running conditions and those when DORIS operated as a synchrotron source. A series of wakefield suppressors (Sec. 3.3) were necessary to reduce the heat load on the target cell. A tungsten collimator (Sec. 3.4) was also housed in the scattering chamber to prevent synchrotron radiation, beam halo, and off-momentum particles from striking the target cell. Finally, an extensive vacuum system (Sec. 3.5) of turbomolecular and Non-Evaporable Getter (NEG) pumps was employed to preserve the vacuum in the DORIS storage ring.

\subsection{Target Cell}

The target cell consisted of an open-ended, elliptical cylinder $(27 \mathrm{~mm}$ horizontal $\times 9 \mathrm{~mm}$ vertical $\times 600 \mathrm{~mm}$ long) made from $0.075 \mathrm{~mm}$ thick aluminum. The elliptical shape was chosen to match the DORIS beam envelope and was set to approximately the $10 \sigma$ nominal horizontal and vertical beam width at the OLYMPUS interaction point to minimize the amount of beam halo striking the cell walls.

The INFN Ferrara group produced several target cells for the OLYMPUS experiment. Cells were formed from two identical stamped sheets of aluminum that were spot-welded together along the top and bottom seams. Each cell was mounted in a frame by a clamp that ran the entire length of the top seam. The frame was made of 6063 aluminum to provide high thermal conductivity at cryogenic temperatures. When installed in the scattering chamber, the cell and frame assembly was suspended from a flange in the top of the scattering chamber (shown in Fig. 3) and its position and orientation could be adjusted. The entire cell and frame assembly was cooled by a cryogenic coldhead. The assembly was wrapped in several layers of aluminized mylar to insulate it from thermal radiation. Without beam or gas flow, the target could reach temperatures below $40 \mathrm{~K}$. During high-luminosity running, a temperature of about $70 \mathrm{~K}$ was sustained.

During operation, hydrogen gas was flowed through the target cell. The hydrogen gas was produced by a commercial hydrogen generator and was controlled by a series of valves, buffer volumes, and mass flow controllers. The gas entered the cell at the center, from a tube that fit snuggly into an opening of the cell's top seam. The gas diffused outwards to the open ends of the cell, where it was removed by the vacuum system. This diffusion was slowed because the hydrogen quickly cooled to the temperature of the cell. The density distribution in the cell was triangular, with peak density at the center of 


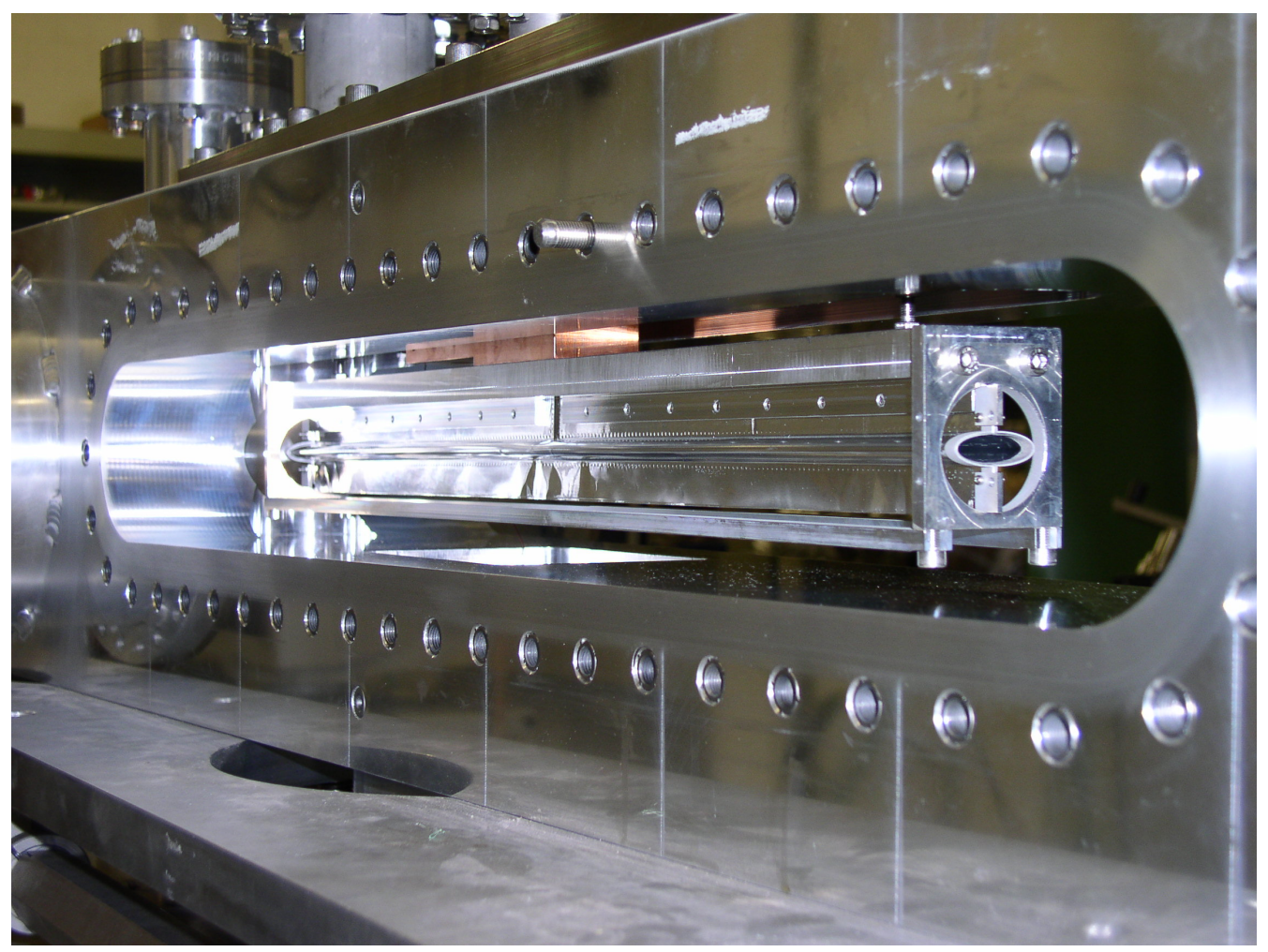

Fig. 3: Photograph of one of the OLYMPUS target cells mounted inside the scattering chamber. 
the cell falling to zero density at either end. A flow rate of $1.5 \times 10^{17} \mathrm{H}_{2}$ molecules per second was required to produce a target thickness of $3 \times 10^{15}$ atoms $\mathrm{cm}^{-2}$.

\subsection{Scattering Chamber}

The OLYMPUS scattering chamber (shown in Fig. 4) was $1.2 \mathrm{~m}$ long and was ma-

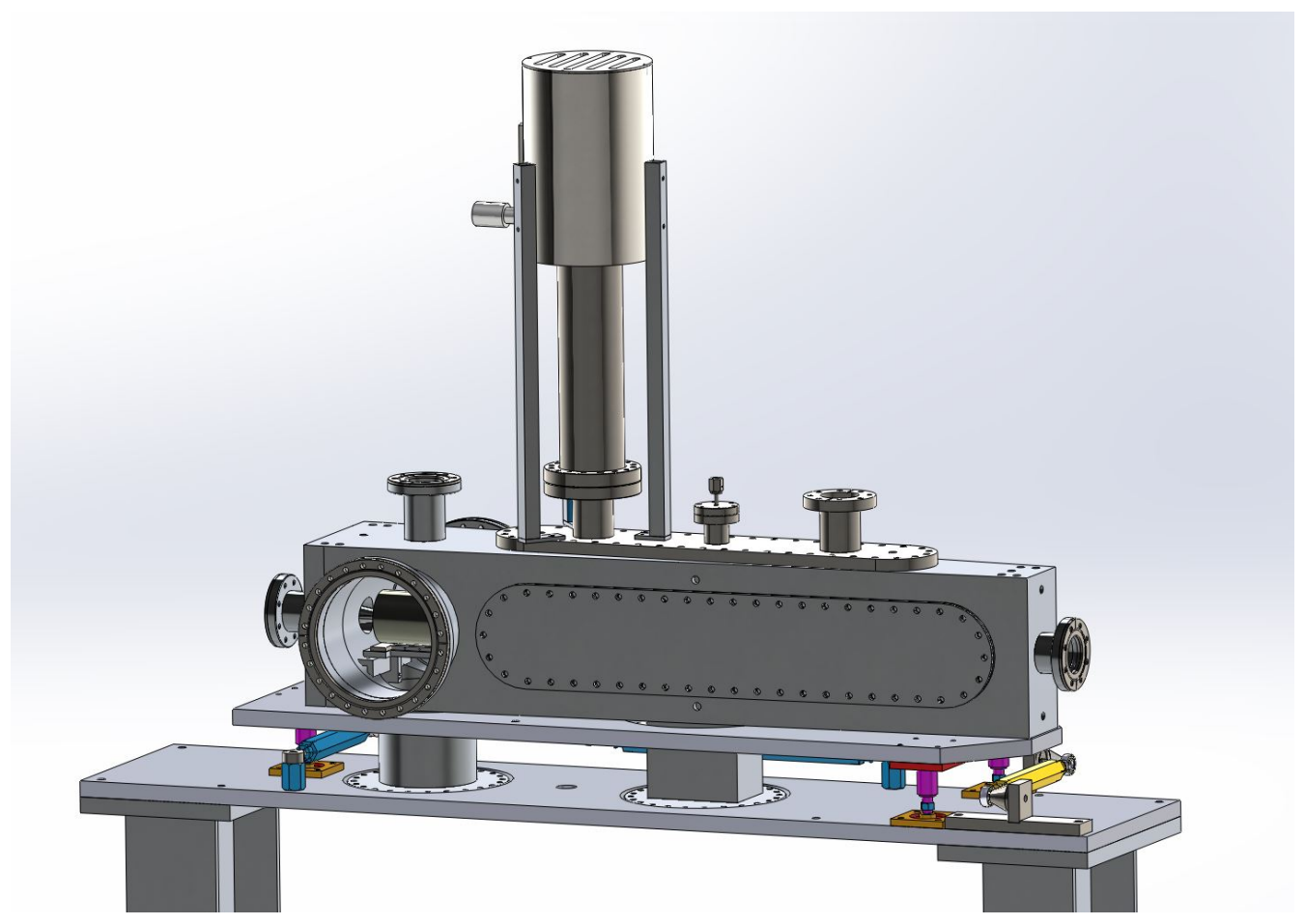

Fig. 4: CAD model of the OLYMPUS scattering chamber.

chined from a solid block of aluminum, with large area windows on the left and right faces. The windows were made of $0.25 \mathrm{~mm}$ thick 1100 aluminum, and nominally subtended a polar angular range of $8^{\circ}$ to $100^{\circ}$ from the center of the target, $6^{\circ}$ to $90^{\circ}$ from $200 \mathrm{~mm}$ upstream, and $10^{\circ}$ to $120^{\circ}$ from $200 \mathrm{~mm}$ downstream. The chamber was trapezoidal in shape to make more of the target cell "visible" to the $12^{\circ}$ detectors.

In addition to windows, the chamber had ports for the beamline (up- and downstream), for pumping (on the bottom surface), for access to the collimator (on the left and right), and for the target cell flange on the top, which had feedthroughs for the hydrogen gas, the coldhead, and various sensors. The main components inside the scattering chamber are shown in Fig. 5

\subsection{Wakefield Suppressors}

Wakefield suppressors were necessary to maintain the target cell at cryogenic temperatures by preventing heating caused by wakefields. The wakefield suppressors consisted 


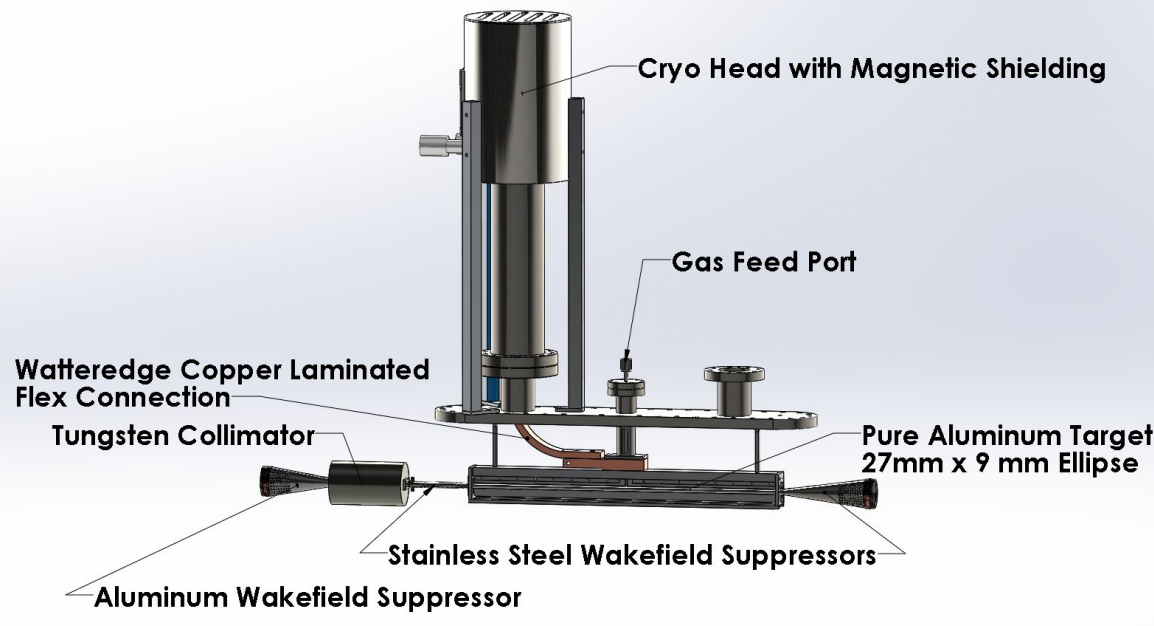

Fig. 5: CAD model of the target cell, wakefield suppressors, and collimator inside the OLYMPUS scattering chamber.

of conducting transitions that were added to fill gaps between conducting structures surrounding the beam. Any sharp transitions or gaps in conductivity would act as electrical cavities that would be excited by the passing beam, creating wakefields and producing heat. To prevent this, three wakefield suppressors were produced to cover the following three transitions:

1. from the circular upstream scattering chamber port (60 $\mathrm{mm}$ in diameter) to the $25 \mathrm{~mm} \times 7 \mathrm{~mm}$ elliptical opening of the collimator,

2. from the exit of the collimator to the entrance of the target cell (both $27 \mathrm{~mm} \times$ $9 \mathrm{~mm}$ ellipses), and

3. from the $27 \mathrm{~mm} \times 9 \mathrm{~mm}$ elliptical exit of the target cell to the circular downstream scattering chamber port (60 $\mathrm{mm}$ in diameter).

With these wakefield suppressors, a target temperature of around $50 \mathrm{~K}$ was maintained during synchrotron operation, and a temperature less than $70 \mathrm{~K}$ was maintained during high-luminosity OLYMPUS running.

The wakefield suppressors were made of stainless steel (except the upstream wakefield suppressor, which was made of aluminum) and plated with silver for improved electrical conductivity. The surfaces were smooth except for many small holes, which were drilled to allow the vacuum system to pump gas through them. The ends of the wakefield suppressors had beryllium-copper spring fingers around their circumference. These spring fingers made sliding connections at an interface that allowed for thermal expansion while maintaining good electrical contact. The upstream wakefield suppressor was 
screwed directly to the collimator making a sliding connection with the upstream scattering chamber port. The other two wakefield suppressors were fixed to rings clamped to the ends of the target making sliding connections to either the downstream scattering chamber port or the collimator. A close-up view of the middle wakefield suppressor is shown in Fig. 6 .

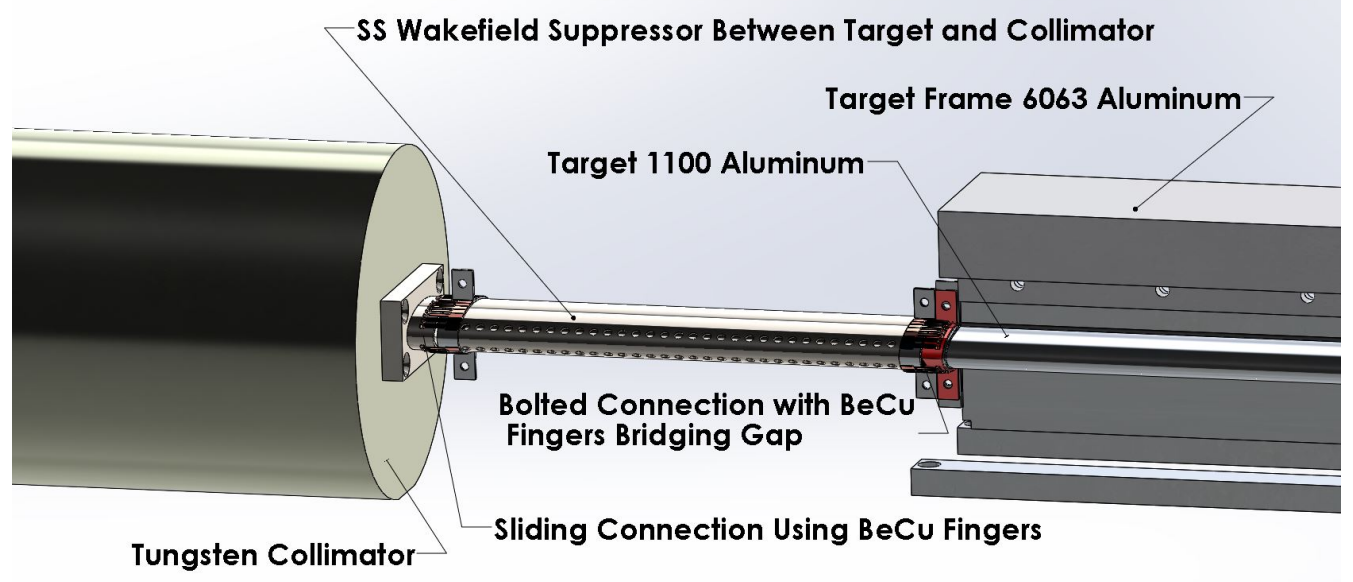

Fig. 6: CAD model of the wakefield suppressor between the collimator and the target cell.

\subsection{Collimator}

Fig. 6 also shows the fixed collimator in front of the target cell. The collimator consisted of a $139.7 \mathrm{~mm}$ long cylinder of tungsten $82.55 \mathrm{~mm}$ in diameter. The outer dimensions were chosen after performing a study on simulated showers of beam-halo particles. It had a tapered elliptical aperture with entrance $25 \mathrm{~mm} \times 7 \mathrm{~mm}$ and exit $27 \mathrm{~mm} \times 9 \mathrm{~mm}$. The collimator was machined from a solid block of tungsten using wire electrical discharge machining 6 . The entrance dimensions were chosen to be slightly smaller than those of the storage cell to shield the target cell walls.

\footnotetext{
${ }^{6}$ Jack's Machine Co. Hanson, MA 02341
} 


\subsection{Vacuum System}

A system of six magnetic levitation turbomolecular pumps (Osakd ${ }^{7}$ TG $1100 \mathrm{M}$ and Edwards $s^{8}$ STP $1003 \mathrm{C}, 800 \mathrm{~L} / \mathrm{s}$ capacity) and NEG pumps (SAES ${ }^{9}$ CapaciTorr CFF $4 \mathrm{H} 0402,400 \mathrm{~L} / \mathrm{s}$ capacity) was used to pump the section of beamline inside the OLYMPUS experiment. This system utilized three stages of pumping to reduce the pressure from the relatively high pressure $\left(\sim 10^{-6}\right.$ Torr $)$ at the scattering chamber (caused by hydrogen gas flowing into the target cell) to the low pressure $\left(\sim 10^{-9}\right.$ Torr) of the DORIS storage ring.

The vacuum system is shown in Fig. 7. Two turbo pumps located in the pit beneath

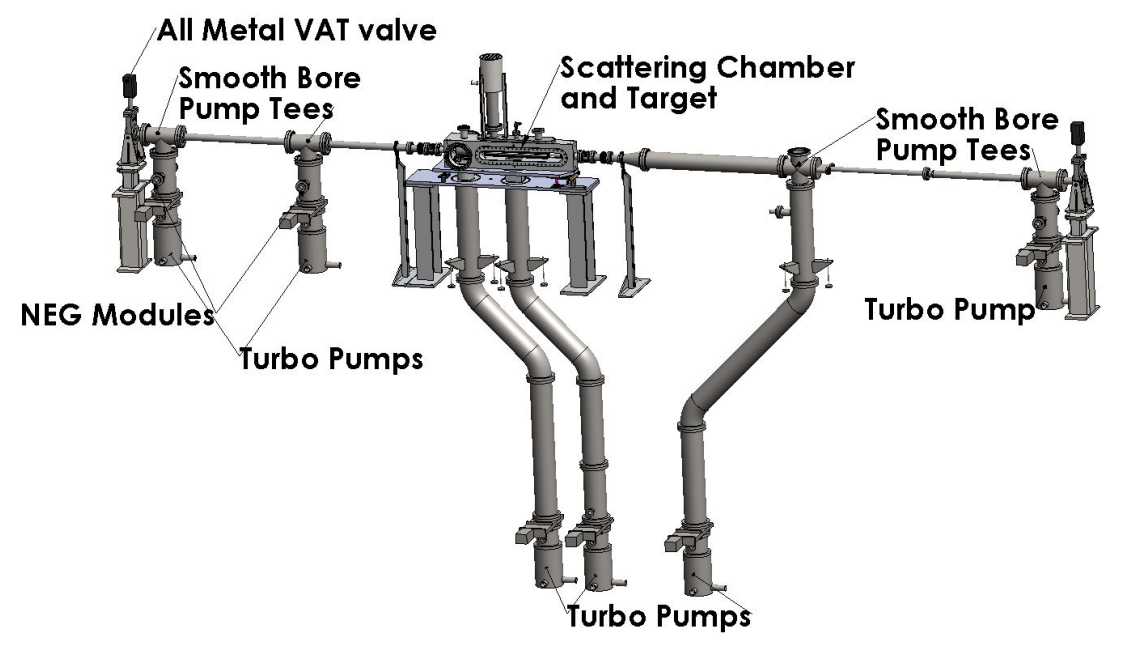

Fig. 7: CAD model of the vacuum system employed for the OLYMPUS experiment.

the experiment were directly connected to the scattering chamber through $200 \mathrm{~mm}$ diameter pipes. Two more turbo pumps were connected to the up- and downstream beamlines approximately $2 \mathrm{~m}$ from the target. At approximately $3 \mathrm{~m}$ from the target another two turbo pumps were used to reduce the pressure in the beamline to the level acceptable for the DORIS storage ring. The four pumping stations furthest from the target also had NEG pumps to improve the pumping of hydrogen.

\section{The OLYMPUS Detector}

The OLYMPUS spectrometer consisted of an eight-coil toroidal magnet with detectors in the two horizontal sectors on either side of the beamline (see Fig. 8). Each of

\footnotetext{
${ }^{7}$ Osaka Vacuum Ltd., Osaka, Japan

${ }^{8}$ Edwards, Crawley, UK

${ }^{9}$ SAES Group, Lainate, Italy
} 


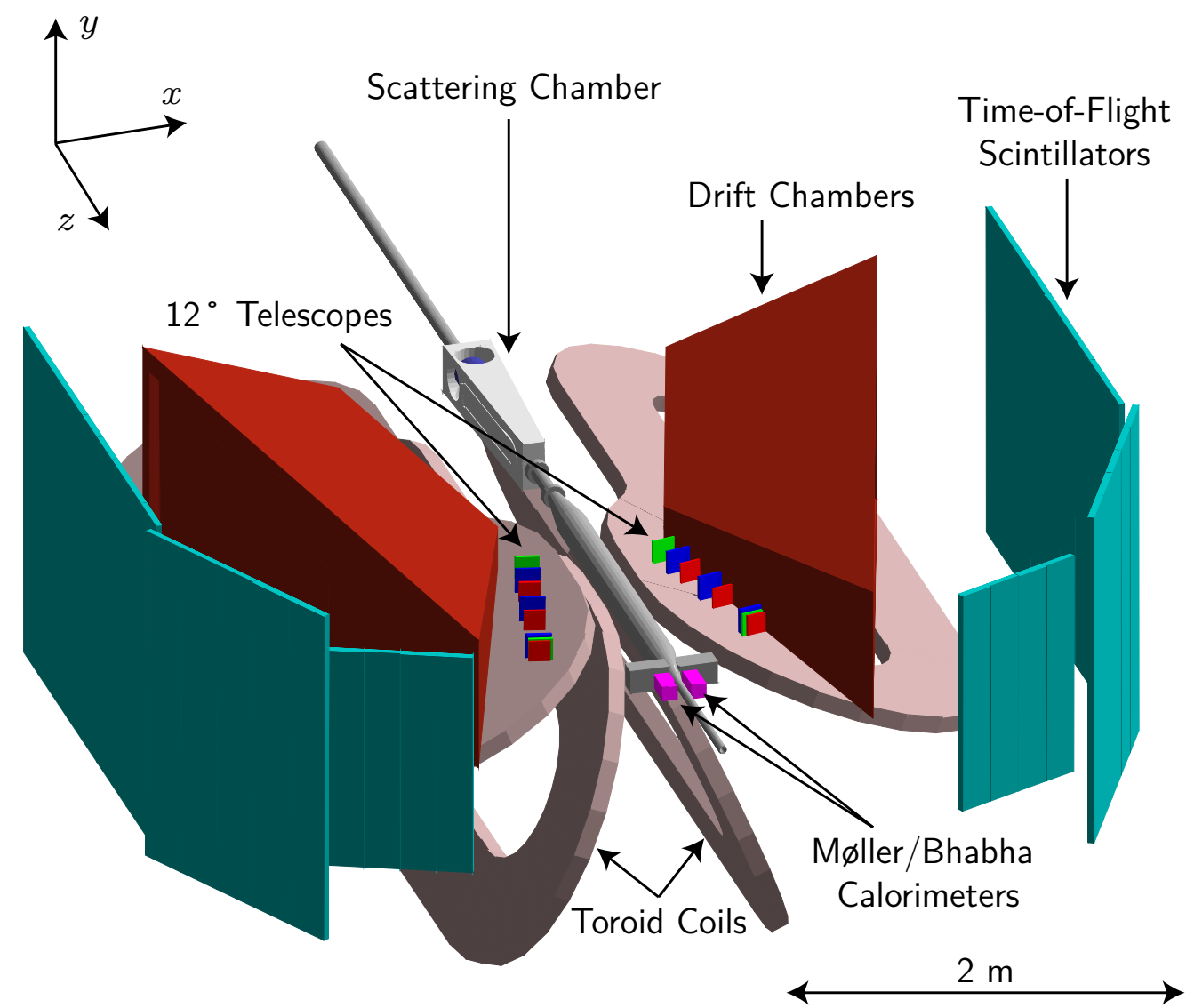

Fig. 8: A solid-model representation of the OLYMPUS detector with the top four magnet coils removed to show the instrumented horizontal sectors. 
these sectors contained drift chambers for particle tracking and an array of time-of-flight scintillator bars for triggering and measurements of energy deposition, particle position, and timing. To monitor the luminosity, OLYMPUS had a redundant system consisting of symmetric Møller/Bhabha (SYMB) calorimeters at $\theta=1.29^{\circ}$ and detector telescopes at $12^{\circ}$ in both sectors, each consisting of three gas electron multiplier (GEM) detectors interleaved with three multi-wire proportional chambers (MWPCs).

The toroidal magnet, drift chambers, time-of-flight detectors, support frames, and many of the readout and control electronics were originally part of the BLAST spectrometer 39 at MIT-Bates. These components were shipped to DESY in spring 2010 where they were reassembled, reconditioned, and modified as necessary for installation in the OLYMPUS detector.

The OLYMPUS experiment was installed in the straight section of the DORIS storage ring, in the location of the former ARGUS experiment 38. The initial assembly took place from June 2010 to July 2011 outside of the DORIS tunnel, to avoid interferring with DORIS operation. The detector was assembled on a set of rails that led (through a removable shielding wall) to the ARGUS site. When the assembly was complete, the shielding wall was removed, the spectrometer was rolled into place in the tunnel, and the wall was rebuilt. The experimental site was $7 \mathrm{~m}$ wide, with a $5 \mathrm{~m}$ deep pit below the beam height. The pit was a convenient location for vacuum pumps, power supplies, and the target gas system because it was deep enough to be outside of the fringes of the magnet field.

In the area outside the shielding wall was an electronics hut, which was supported on the same set of rails. The hut housed the detectors' readout and control electronics, the high voltage supplies, and the computer systems. The electronics hut could be accessed even when the DORIS beam was circulating.

The following sections describe the detector components in greater detail.

\subsection{Toroidal Magnet}

The toroidal magnet consisted of eight copper coils placed around the beam line and scattering chamber so that the beam traveled down the toroid's symmetry axis (see Fig. 9). The coils divided the space around the beamline into eight sectors. The two sectors in the horizontal plane were instrumented with detectors. During normal operation, the magnet produced a field of about $0.28 \mathrm{~T}$ in the region of the tracking detectors.

The magnet was originally designed and used for the BLAST experiment, and has been described in a previous article [40. The choice of a toroidal configuration for BLAST was made to ensure a small field along the beamline in order to minimize any effects on a spin-polarized beam and to limit field gradients in the region of the polarized target. Since OLYMPUS used neither a polarized beam nor a polarized target, these concerns were not as important. However, during the initial set-up, the magnetic field along the beamline was measured and the coil positions adjusted to achieve an integrated field $<0.005 \mathrm{~T} \cdot \mathrm{m}$ to avoid perturbing the beam's position or direction.

Each of the toroid's eight coils consisted of 26 turns of 1.5 inch square copper tubes, organized into two layers of 13 turns. A circular hole, 0.8 inches in diameter, ran down the length of each tube and served as a conduit for cooling water. During assembly, the tubes were individually wrapped with fiberglass tape and then collectively potted in an 


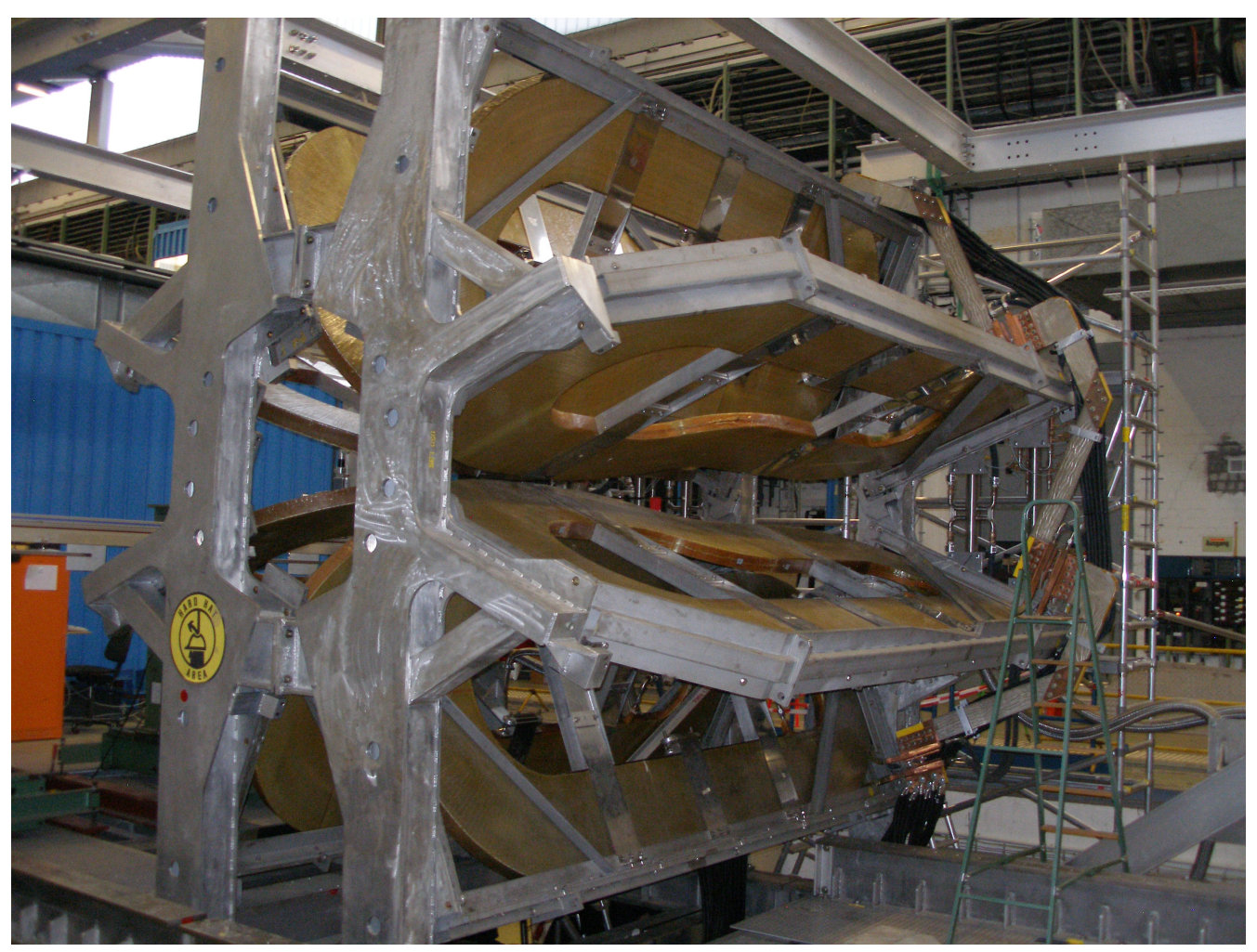

Fig. 9: The toroid magnet assembled at DESY before the subdetectors were installed

epoxy resin matrix. The final outline and nominal position relative to the beam line and target center at the coordinate origin are shown in Fig. 10. The coils are narrower at one end to accommodate the scattering chamber and wider at the other to extend the high-field region to more forward angles, where scattered particles have higher momenta.

The magnetic field served two purposes. The first was to bend the tracks of charged particles, allowing their momentum and charge sign to be determined from the curvature of their tracks. The second was to sweep away low-energy, charged background particles from the tracking detectors. Though a stronger magnetic field would have improved momentum resolution and reduced the background, it would also have increased the Lorentz angle of drift electrons in the tracking detectors, making track reconstruction more difficult. A balance was struck by choosing a current of $5000 \mathrm{~A}$ for normal operation, which produced a field of about $0.28 \mathrm{~T}$ in the high-field regions.

Originally, it was planned to alternate the polarity of the magnet every few hours to reduce systematic uncertainties. However, this proved impractical at high luminosity. In the negative polarity setting, the magnet bent negatively charged particles outward from the beamline. The drift chambers were hit with a large background of low-energy electrons, which frequently caused the high voltage supply to exceed its current threshold and trip. Attempts to adequately shield the drift chambers, both by adding material and by increasing the magnetic field strength, were unsuccessful. Consequently, the negative 


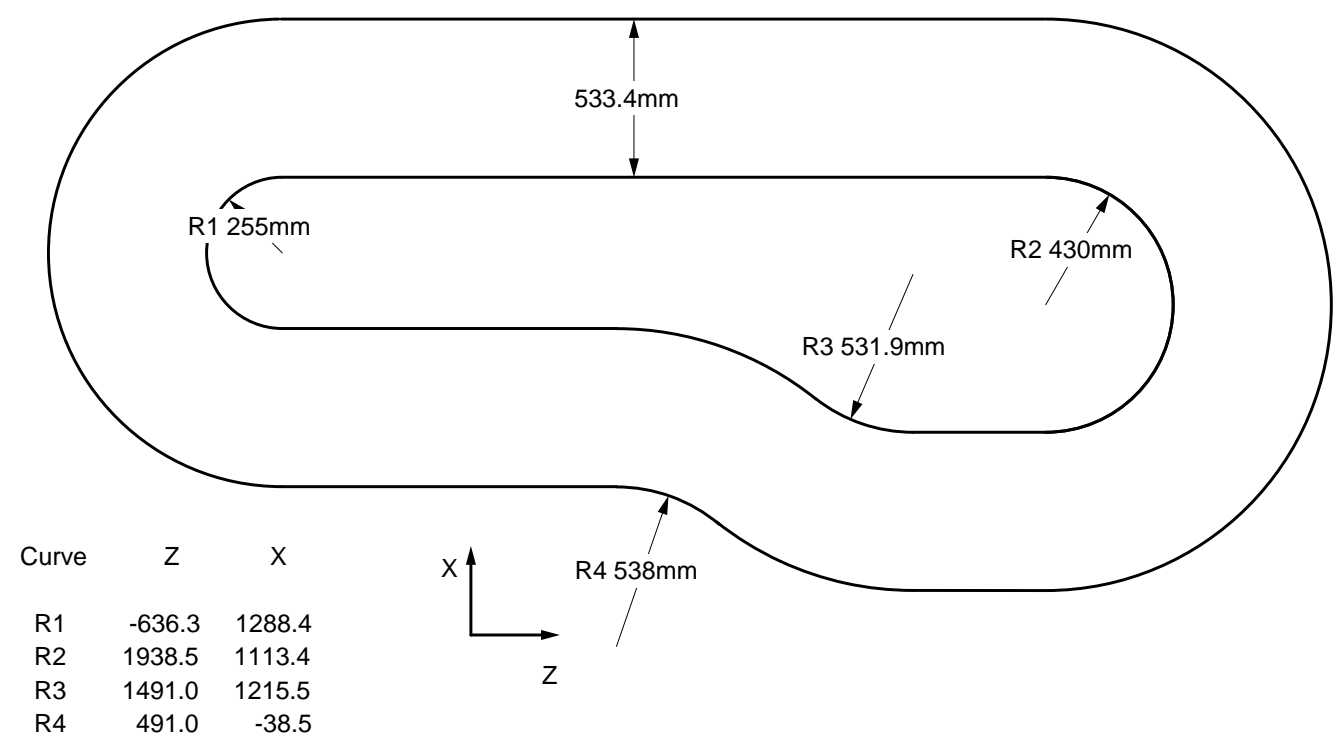

Fig. 10: Planar view of BLAST coil outline showing dimensions and position relative to the center of the target cell.

polarity setting was limited to low-luminosity running, and only about $13 \%$ of the total luminosity was collected in this mode. The limited negative polarity data will provide a check on systematic uncertainties.

After the experimental running period was completed, the detectors and downstream beamline were removed in order to conduct a measurement of the magnetic field. By convention, the direction of the beam was labeled as the OLYMPUS $z$-axis, the $y$-axis pointed up, and the $x$-axis pointed toward the left sector, forming a right-handed coordinate system. The field region was scanned using a 3D Hall probe mounted to a rod, driven by several translation tables. The rod was mounted to a long XYZ table with a range of motion of $0.2 \mathrm{~m} \times 0.2 \mathrm{~m} \times 6 \mathrm{~m}$. This long table was supported by two large XY tables that augmented the $x$ and $y$ ranges each by $1 \mathrm{~m}$. The range of motion was further extended in $x$ by substituting rods of different lengths and in $y$ by adding a vertical extension piece. The apparatus was used to measure the field over a grid of points on the left sector, before being transported and reassembled for a similar measurement of points on the right sector. The grid extended from $-0.5 \mathrm{~m}$ to $3.5 \mathrm{~m}$ in $z$. In $x$ and $y$, the grid was limited to the triangular space between the coils, but extended to $\pm 2.7 \mathrm{~m}$ on either side of the beamline. The grid points were spaced $0.05 \mathrm{~m}$ apart in the region within $1 \mathrm{~m}$ of the beamline, and $0.10 \mathrm{~m}$ apart in the outer region, where the field changed less rapidly. In total, approximately 35,000 positions were measured over a two month period, including the downstream beamline region, which was measured redundantly from the left and the right.

After the initial setup of the apparatus, the precise position of the XYZ tables was measured with a laser tracking station over the course of a typical scan in $z$. This showed that the Hall probe position varied in $x$ and $y$ as a function of $z$ during a scan, but that 
the shape was quite reproducible. To correct for this variation, the start and end points of each scan were measured using a theodolite and a total station. This data then allowed the position of the Hall probe to be determined for each measurement. Position-corrected data for the vertical component of the field are shown in Fig. 11.

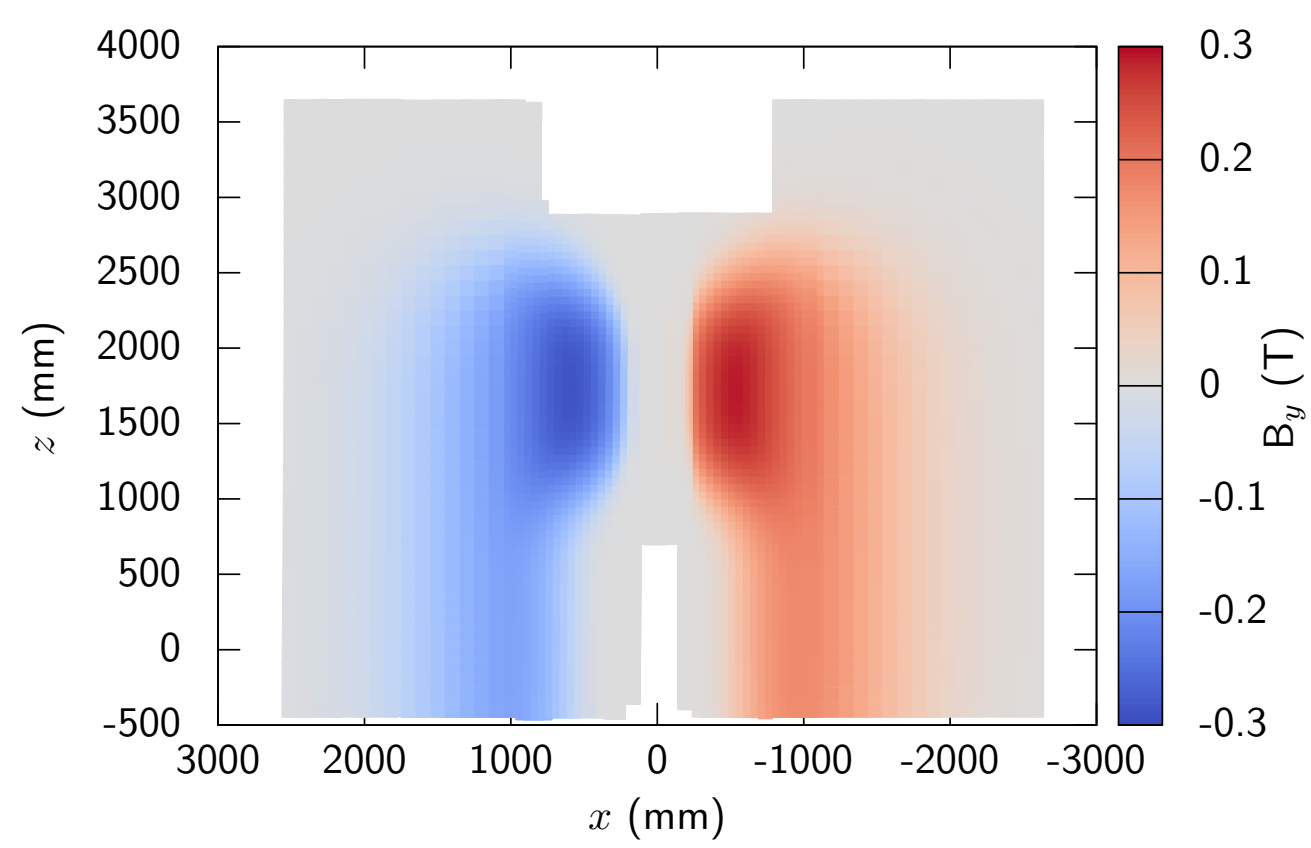

Fig. 11: Measurements of the vertical magnetic field component $B_{y}$ in the horizontal plane as viewed from above

After correcting the Hall probe positions, a fit was performed to the magnetic field data. The fit was based on a model of the coil geometry with a Biot-Savart calculation of the magnetic field. The fit allowed the coil positions to vary slightly to best match the measurements. This model was then used to extrapolate the field over the entire volume around the OLYMPUS detector for use in track reconstruction and in the OLYMPUS Monte Carlo simulation.

\subsection{Drift Chambers}

The drift chambers used for the OLYMPUS experiment came from the BLAST experiment at MIT-Bates and have been described in great detail elsewhere [39], so the following description will be brief while mentioning new and updated features.

The drift chambers were used to measure the momenta, charges, scattering angles, and vertices of out-going charged particles. The drift chambers had a large angular acceptance, subtending a range of $20^{\circ}-80^{\circ}$ in polar angle and $\pm 15^{\circ}$ in azimuth. The chambers were oriented to be normal to a polar angle of $73.54^{\circ}$. Because of these choices, the chambers were trapezoidal in shape (see Fig. 12).

The drift chambers were arranged in two sectors that were positioned on either side of the target, in the horizontal plane. Each sector contained three drift chambers (inner, 


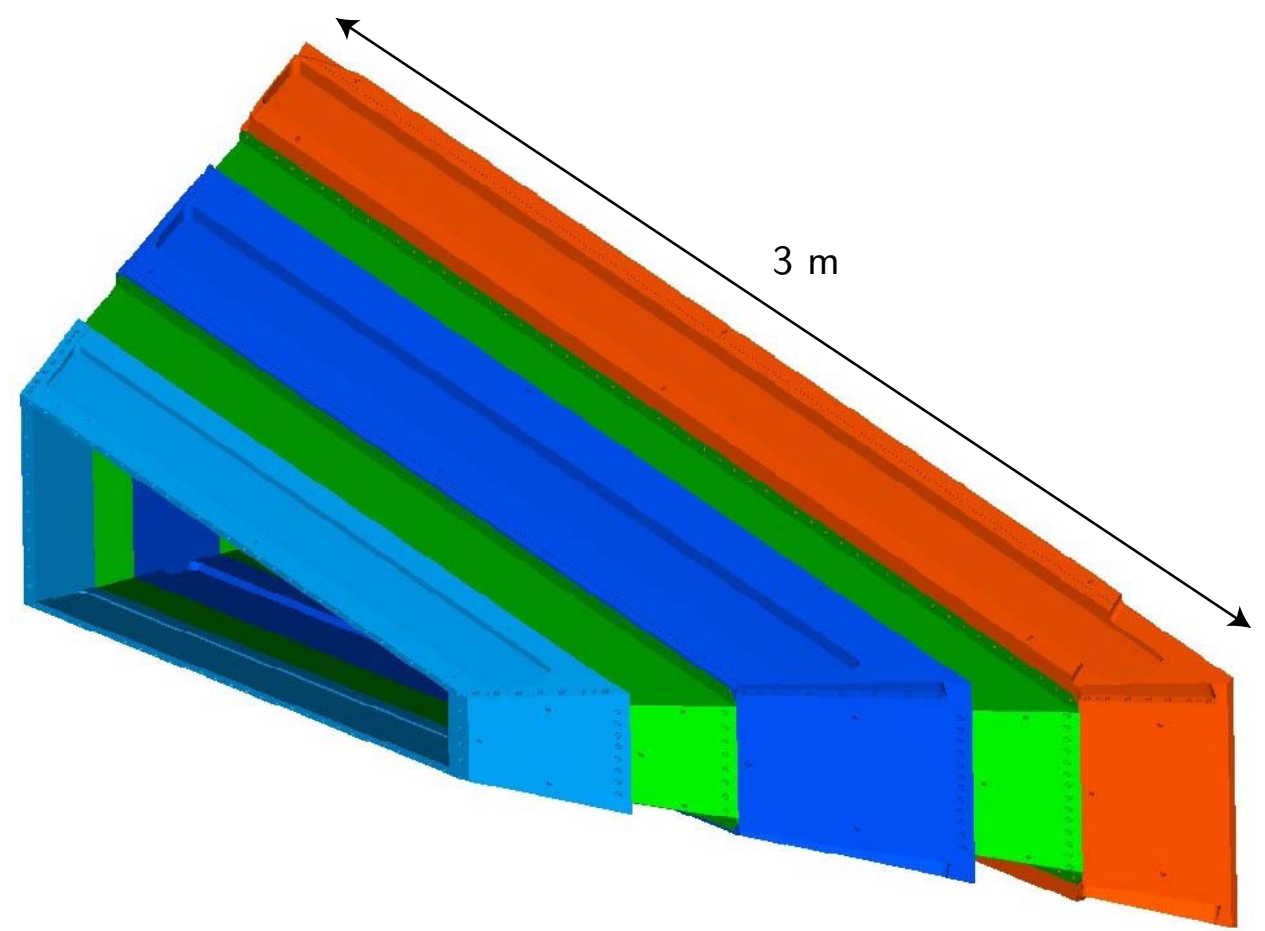

Fig. 12: Isometric view of all three drift chambers assembled into a single gas volume. 
middle, and outer) joined together by two interconnecting sections to form a single gas volume. Thus, only one entrance and one exit window were needed, reducing multiple scattering and energy loss. The drift chambers combined had approximately 10,000 wires, which were used to create the drift field. Of these, 954 were sense wires, which read out the signals from ionization caused by a charged particle track.

Each chamber consisted of two super-layers (or rows) of drift cells, with $20 \mathrm{~mm}$ separation between the super-layers. The drift cells were formed by wires in a "jet style" configuration. Fig. 13 shows a cross-sectional view of a portion of one chamber with the
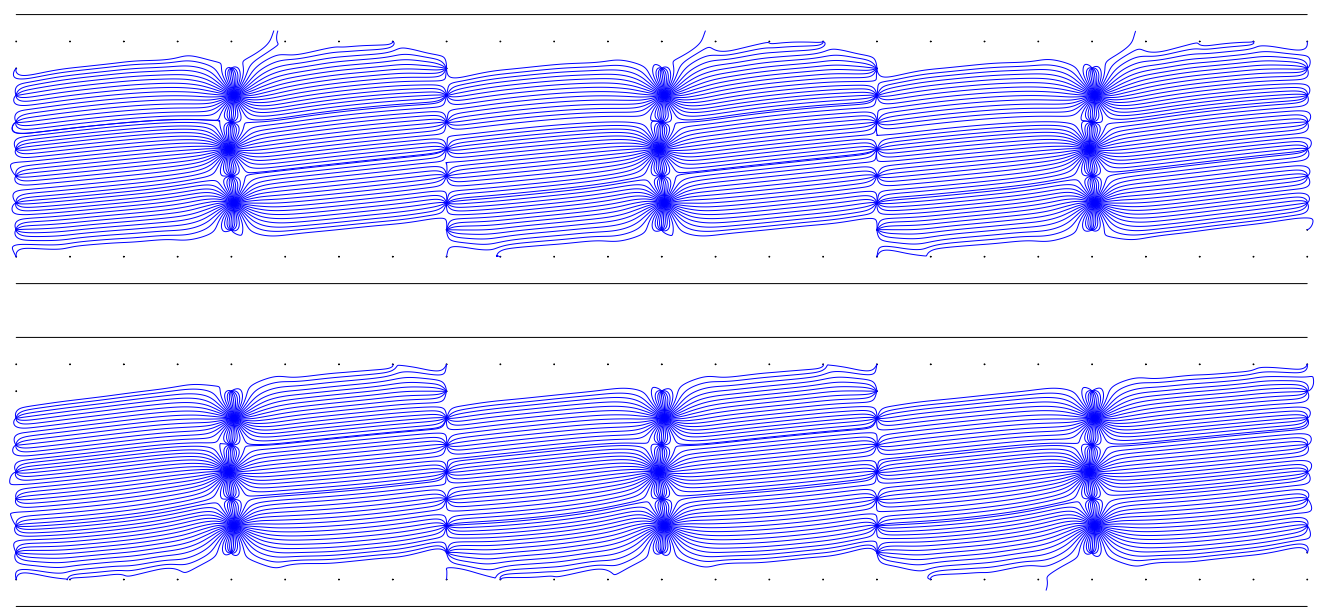

Fig. 13: Portion of a chamber showing the two super-layers of drift cells formed by wires. Lines of electron drift in the drift cells assuming a typical magnetic field around $3.0 \mathrm{kG}$ are also shown.

two super-layers of drift cells. It also shows characteristic "jet-style" lines of electron drift in a magnetic field. Each drift cell was $78 \times 40 \mathrm{~mm}^{2}$ and had three sense wires staggered $\pm 0.5 \mathrm{~mm}$ from the center line of each cell to help resolve the left/right ambiguity in determining position from the drift time. The wires in one super-layer were strung with a $10^{\circ}$ stereo angle relative to wires of the other so that each chamber could localize a trajectory in three dimensions.

Because transporting the chambers in a way that would protect the wires from breaking was infeasible, the chambers were completely rewired in a clean room at DESY over a period of about three months during the summer of 2010. In addition to new wires, improvements were made to the front-end electronics, building on experience gained from BLAST.

For the experiment, an Ar: $\mathrm{CO}_{2}: \mathrm{C}_{2} \mathrm{H}_{6} \mathrm{O}$ gas mixture (87.4:9.7:2.9) was chosen for the drift chambers. The ethanol was added by bubbling an $\mathrm{Ar}: \mathrm{CO}_{2} \quad(90: 10)$ gas mixture through a volume of liquid ethanol kept near $5 \mathrm{C}$. The chambers were maintained at a pressure of approximately 1 inch of water above atmospheric pressure with a flow rate of around $5 \mathrm{~L} / \mathrm{min}$.

Signals in the sense wires were processed with front-end electronics housed in the recesses of the interconnecting sections before being sent to TDC modules in the electronics 
hut. The signals were first decoupled from the high-voltage on new, custom-designed, high-voltage distribution boards. The signals next passed to Nanometrics System: 10 N-277L amplifier/discriminators. Then the signals were passed by Ethernet cable to the electronics hut, to LeCroy ${ }^{11} 1877$ Multihit TDC modules, operated in common-stop mode, with the stop signal being provided by a delayed trigger signal. The digitized signals were read out by the data acquisition system. An example TDC spectrum for a single wire is shown in Fig. 14.

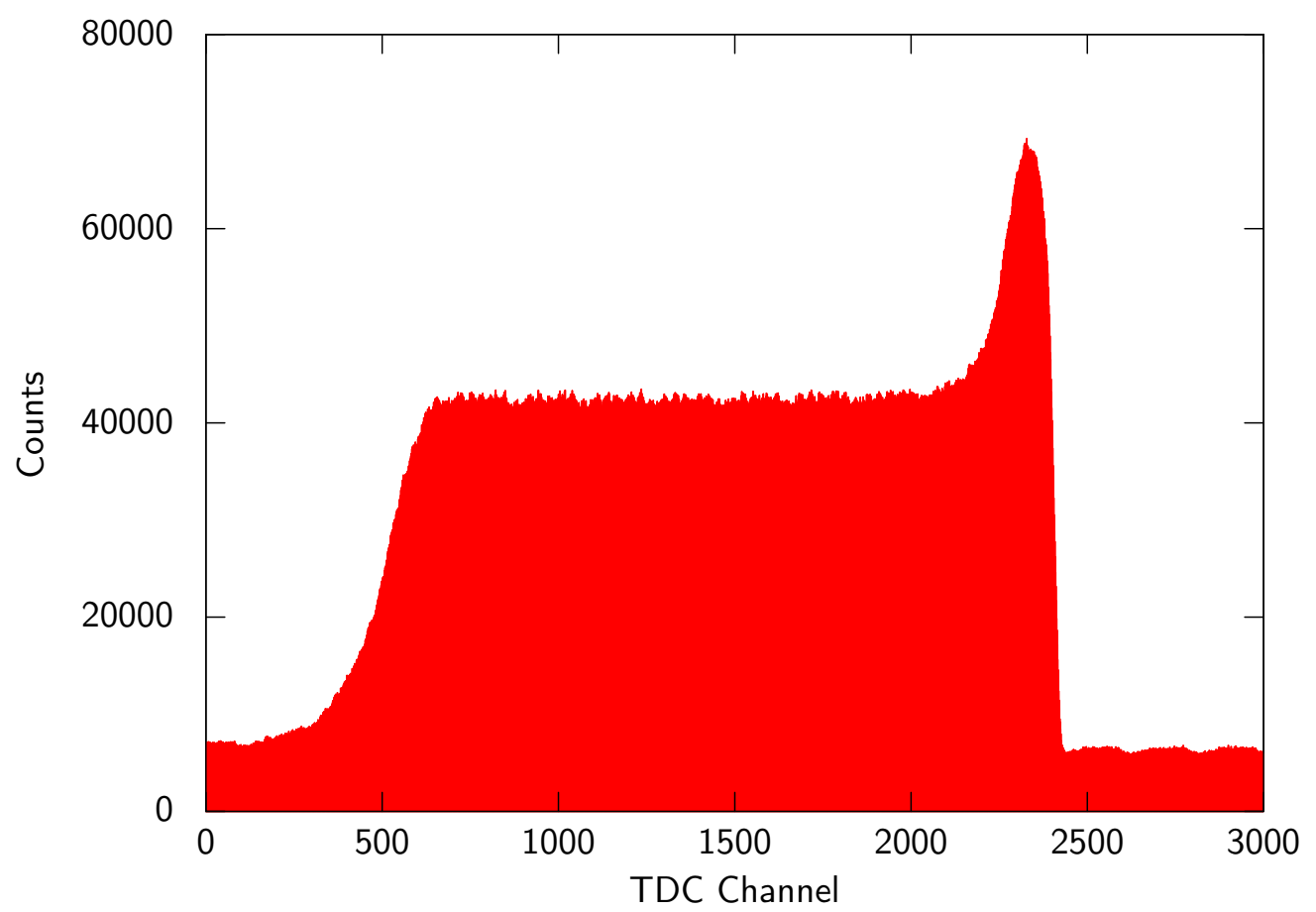

Fig. 14: A typical TDC spectrum for a single wire has a "church shape," which is characteristic of jet-style drift chambers in common-stop mode.

\subsection{Time-of-Flight Detectors}

The time-of-flight (ToF) detector was adapted from the system used for the BLAST experiment 39. Each sector consisted of 18 vertical scintillator bars read out with photo-multiplier tubes (PMT) mounted at both ends, as shown in Fig. 15.

The four most-forward bars on each side were $119.4 \mathrm{~cm}$ high, $17.8 \mathrm{~cm}$ wide, and $2.54 \mathrm{~cm}$ thick. The remaining 14 bars on each side were $180.0 \mathrm{~cm}$ high, $26.2 \mathrm{~cm}$ wide, and $2.54 \mathrm{~cm}$ thick, so as to cover the entire acceptance of the drift chambers. The Glasgow University group designed and constructed a new support structure which allowed a tight

${ }^{10}$ Nanometric Systems, Berwyn, IL, USA

${ }^{11}$ Teledyne Lecroy, Chestnut Ridge, NY, USA 


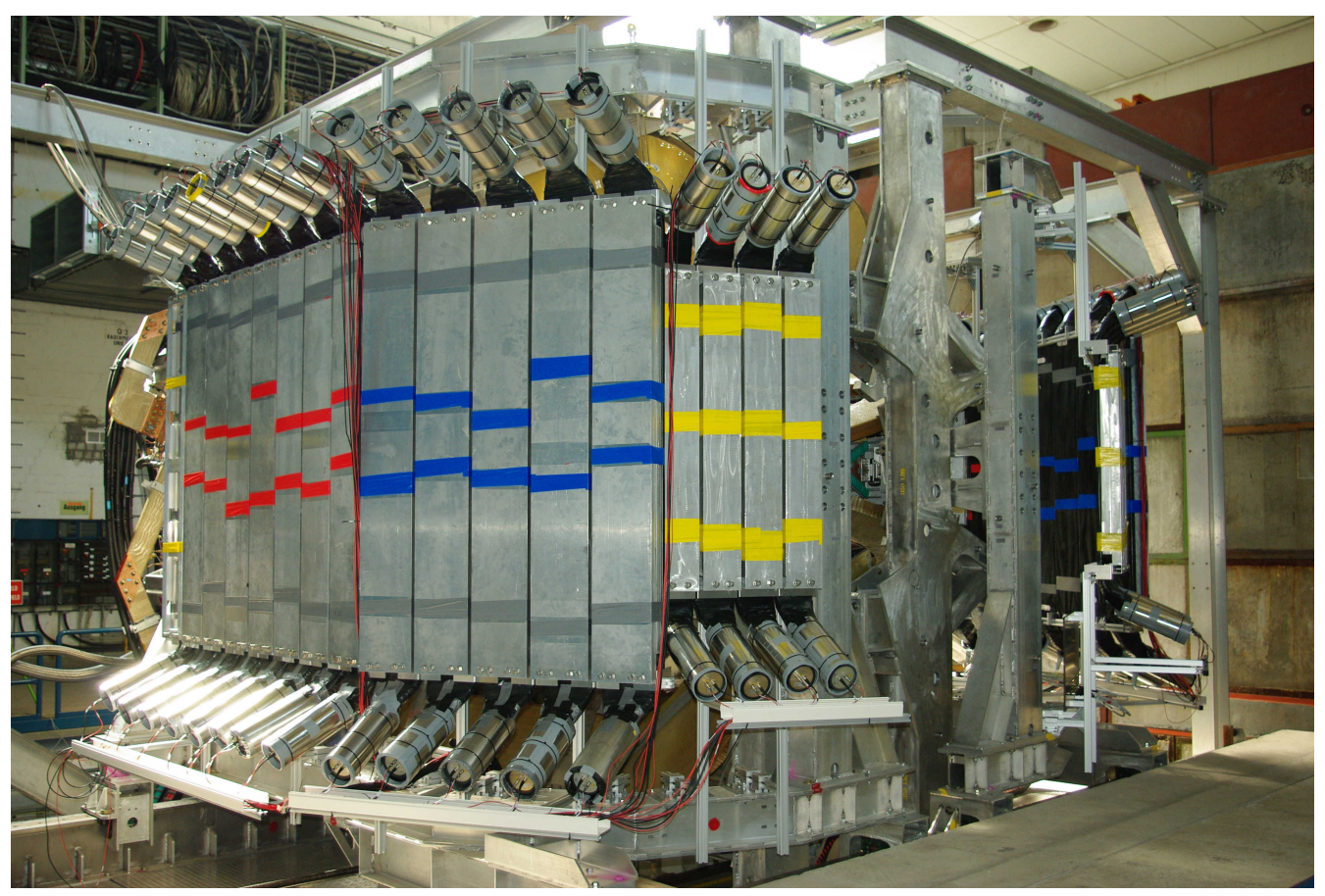

Fig. 15: Photograph of the mounted ToF detectors during assembly of the OLYMPUS detector. 
arrangement and quick replacement of individual bars. The bars were arranged in three planar sections oriented with their normal approximately pointing toward the target area. The rearmost two bars in each sector were not present in BLAST and were added to expand the acceptance of OLYMPUS at large $\theta$.

The ToF detector provided the timing signals used to trigger the readout and data acquisition system for the majority of detector components. In particular, it provided the common-stop signal for the drift chamber TDCs. The main trigger logic of the experiment required the presence of at least one top/bottom coincidence in both sectors (see Sec. 6.1). The ToF PMT signals were processed through passive splitters and recorded by both TDCs and ADCs. The analog PMT signals were discriminated with constant fraction discriminators for the forward 16 bars on each side, and with leadingedge discriminators for the rearmost two bars. The logic signals were further processed for the trigger which in turn provided the common-start signal for the ToF TDCs and the common-stop signal for the drift chamber TDCs. The differential splitter outputs were connected to integrating ADCs. The integrated signal from a given bar provided an estimate of the energy deposited in the bar, while the relative time difference between the top and bottom tube signals from a bar provided a rough measurement of the hit position. The mean signal times of the top and bottom signals were approximately independent of the hit position. The difference in mean times between pairs of ToF bars in opposite sectors measured the difference in time-of-flight between scattered and recoiling particles for interactions originating in the target or measured the time-of-flight of cosmic ray particles traversing the detector.

The active volume of the ToF bars consisted of Bicron ${ }^{12}$ BC-408 plastic scintillator, chosen for its fast response time (0.9 ns rise time) and long attenuation length $(210 \mathrm{~cm})$. At the ends of each bar, the sensitive volumes were connected via Lucite light guides to 3-inch diameter Electron Tubes $\$^{13}$ model 9822B02 photomultiplier tubes equipped with Electron Tubes EBA-01 bases. The PMT signals exhibited a typical amplitude of about $0.8 \mathrm{~V}$ with a rise time of a few nanoseconds. The light guides were bent away from the interaction region to orient the PMTs roughly perpendicular to the toroidal magnetic field. Additionally, each PMT was encased with mu-metal shielding. Due to these measures, the toroidal magnetic field had no discernible effect on the ToF gains. Each PMT base utilized actively-stabilized voltage dividers to avoid variation of signal timing with gain.

Due to aging and radiation damage, some of the scintillator bars were found to have short attenuation lengths. This was determined by examining the TDC and ADC signals for each bar. Problematic bars were replaced before data taking.

After the experiment, during the cosmic ray runs, the efficiencies for top/bottom coincidences were measured by sandwiching the center region of each bar with a pair of small test scintillators. These tests found efficiencies to be around $96-99 \%$ for signals originating near the center of each bar as shown in Fig. 16

\footnotetext{
${ }^{12}$ Bicron, Solon, OH, USA

${ }^{13}$ Electron Tubes Ltd, Ruislip, Middlesex, England
} 

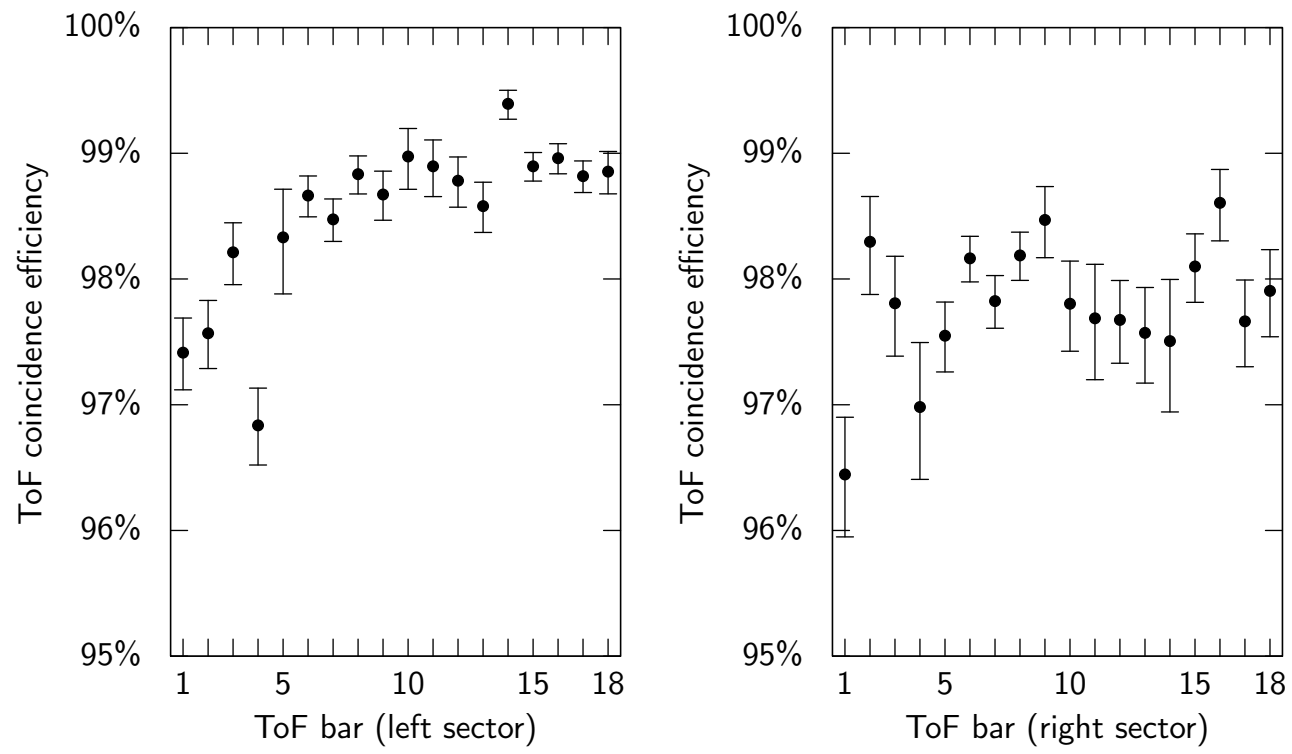

Fig. 16: Efficiencies for each TOF scintillator bar determined during the cosmic running period.

\section{Luminosity Monitors}

The physics goals of OLYMPUS required the very precise and accurate measurement of the ratio of the integrated luminosities with positron and electron beams delivered to the experiment. To achieve this, OLYMPUS included three systems to measure the luminosity redundantly:

- The slow control system (Sec. 7) monitored the beam current and gas flow to the target. The system additionally used measurements of the target cell temperature, in conjunction with the known cell geometry, to compute the target density and thickness during running. The product of the target thickness and beam current was corrected for the deadtime of the data acquisition system to produce a first estimate of the instantaneous luminosity.

- The $12^{\circ}$ luminosity monitors (Sec. 5.1) measured elastically scattered leptons in a small angular range in coincidence with the recoil proton detected in the opposite sector drift chamber. Each monitor consisted of a telescope of three gas electron multiplier (GEM) detectors (Sec. 5.1.1) interleaved with three multi-wire proportional chambers (MWPCs) (Sec. 5.1.2). At $\theta=12^{\circ}$ the two-photon contribution to elastic scattering is expected to be negligible, the known ep elastic cross section can be used to provide a luminosity measurement. The system was designed to provide a luminosity measurement with a statistical precision better than $1 \%$ each hour.

- A high precision measurement using symmetric Møller and Bhabha scattering was implemented using $\mathrm{PbF}_{2}$ calorimeters placed symmetrically at $\theta=1.29^{\circ}$ in the left and right sectors (Sec. 5.2. Comparing the observed $e^{-} e^{-}$and $e^{+} e^{-}$elastic 
scattering rates with the known Møller and Bhabha cross sections provided a means of measuring of the luminosity for each beam species with very high statistical precision in very short time frames.

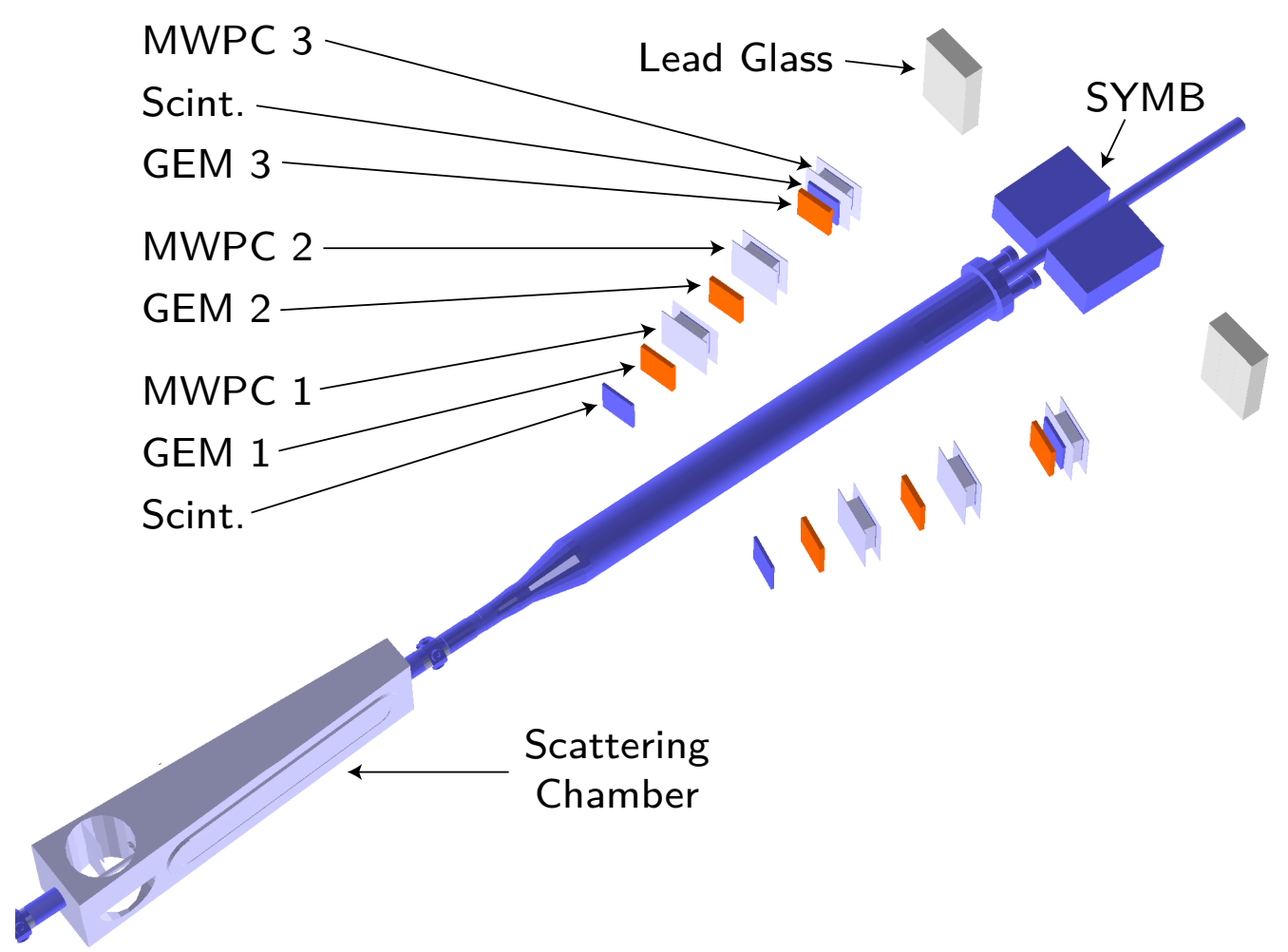

Fig. 17: Layout of the $\theta=12^{\circ}$ luminosity monitors and the symmetric Møller/Bhabha calorimeters

Fig. 17 provides a schematic overview of the $12^{\circ}$ and symmetric Møller/Bhabha luminosity monitoring systems.

\subsection{The $12^{\circ}$ Luminosity Monitoring System}

The $12^{\circ}$ luminosity monitoring system consisted of two telescopes, each composed of three GEM and three MWPC detectors. A pair of thin scintillators with silicon photomultiplier (SiPM) readout contributed to the trigger. The telescopes tracked leptons scattering through small angles, a region where the asymmetry between electron and positron scattering was expected to be small. The telescopes were mounted to rails on the forward faces of the drift chambers to fit in the space between the toroid coils on each side of the beamline. In this position, the telescopes had a clear view of most of the target cell. The two types of detectors provided redundancy for a high efficiency measurement as well as a cross check against systematic effects. A photograph of one of the $12^{\circ}$ telescopes is shown in Fig. 18. 


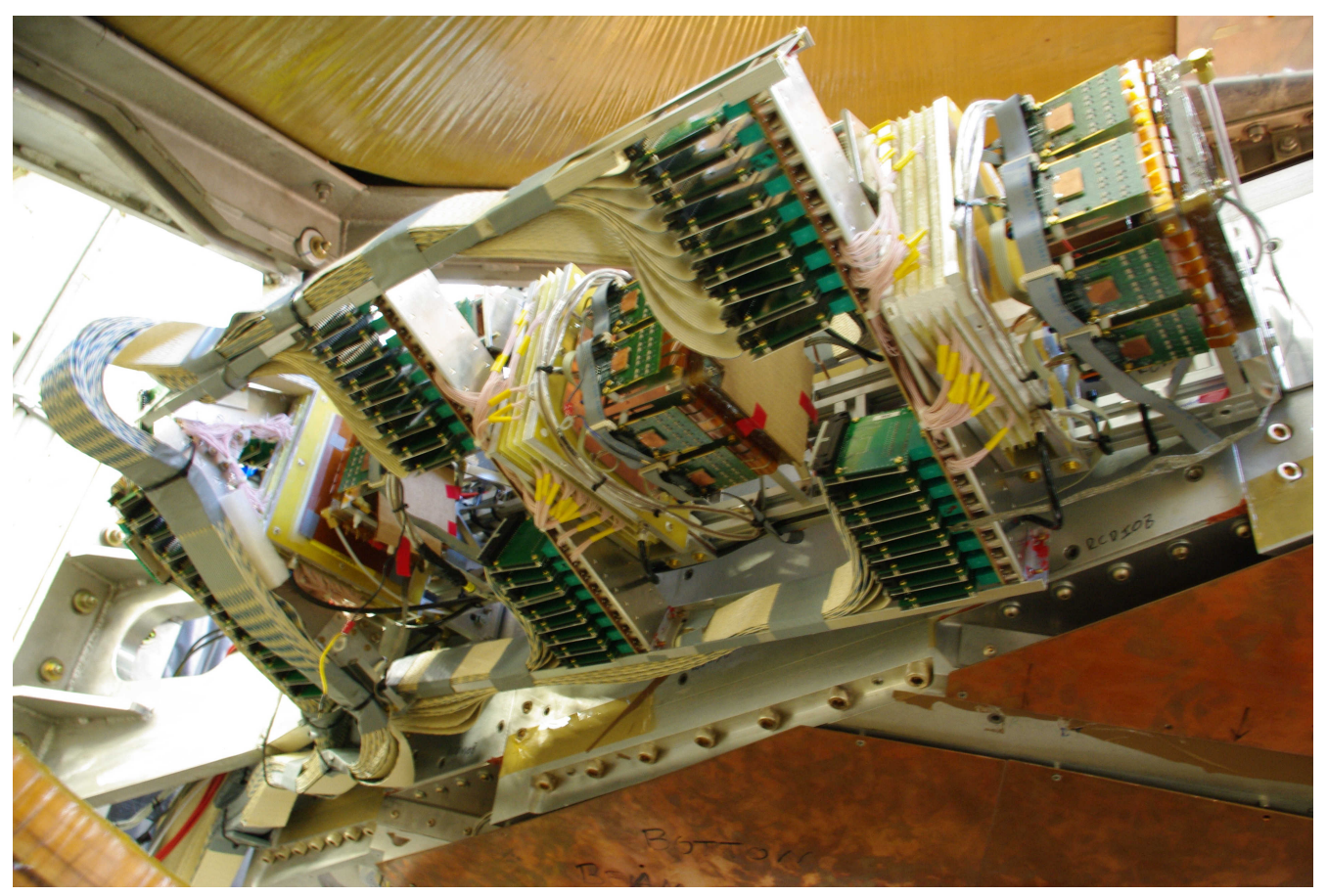

Fig. 18: Photograph of one of the $12^{\circ}$ GEM/MWPC telescopes.

\subsection{1. $12^{\circ}$ GEM Detectors}

The triple-GEM detectors with 2D strip readout were designed at the MIT-Bates Linear Accelerator Center and were constructed at Hampton University. INFN Rome provided the front-end and readout electronics for the GEMs, which were designed in collaboration with INFN Genoa. Each individual GEM chamber was constructed as a stack of frames and foils glued together (see Fig. 19p). Each stack included a readout board with three GEM foils and a cathode foil. Two pressure volume foils enclosed the gas volume to avoid deforming the readout or cathode foils had these been used to enclose the gas volume. There was a $2 \mathrm{~mm}$ space between each GEM foil and between the last GEM foil and the readout board. The pressure volume foils and the cathode foils were spaced $3 \mathrm{~mm}$ from the adjacent foils. All of the components were tested individually before they were assembled into a detector. All of the electrical and gas connections were accessible on the edges of the stack, or in special cutouts in the case of the high voltage connections. A simple resistive voltage divider card provided the high voltage to all foils. A premixed, Ar: $\mathrm{CO}_{2}$ 70:30 gas mixture was used.

The GEM, cathode, and readout foils were manufactured by TechEtch ${ }^{14}$. Each GEM foil consisted of $50 \mu \mathrm{m}$-thick Kapton clad on both sides with $5 \mu \mathrm{m}$-thick layers of copper. The GEM foils were chemically etched to produce $70 \mu \mathrm{m}$ holes in an equilateral triangular pattern with $140 \mu \mathrm{m}$ pitch over the active area of the detector (approximately

${ }^{14}$ TechEtch Inc. Plymouth, MA 


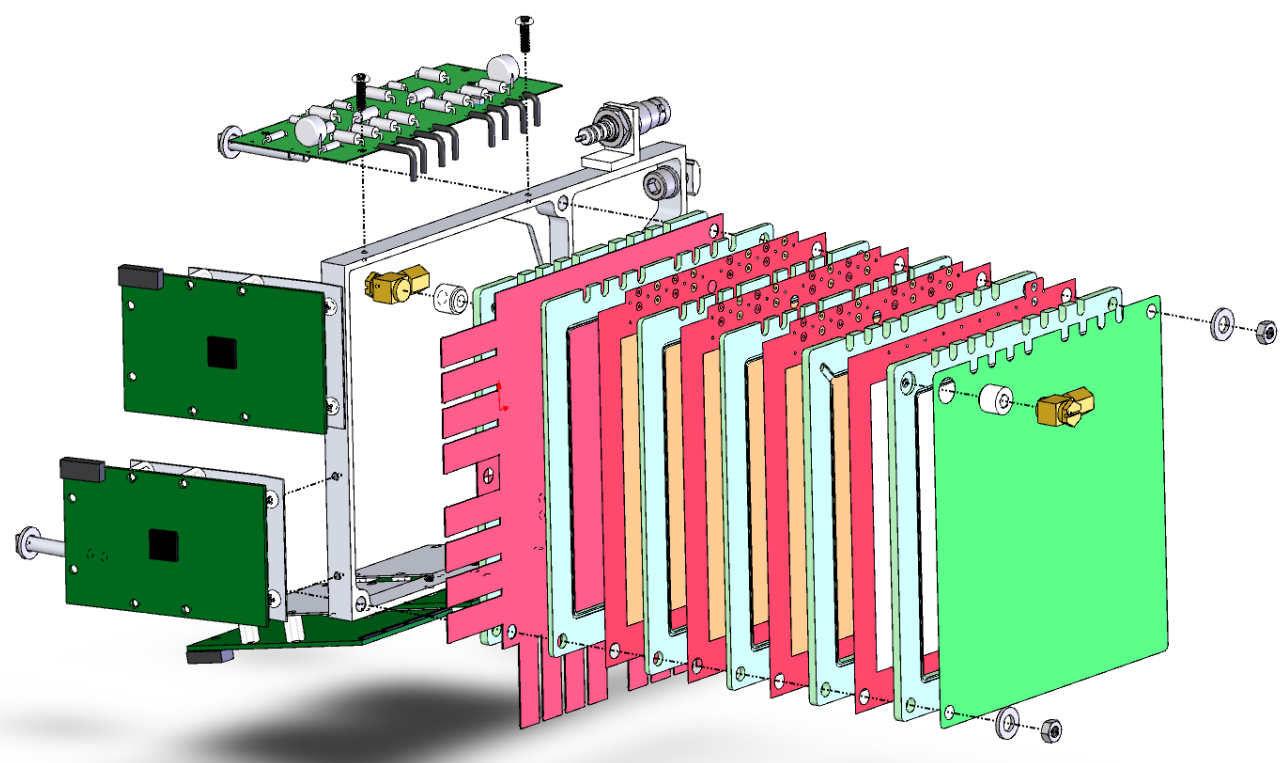

Fig. 19: An exploded view of a single triple-GEM detector. 
$10 \mathrm{~cm} \times 10 \mathrm{~cm}$ ). The cathode foil consisted of $50 \mu \mathrm{m}$-thick Kapton clad on only one side with a $5 \mu \mathrm{m}$-thick layer of copper and no holes. The cathode foil provided a uniform electric field throughout the primary ionization area. The pressure volume foils consisted of $50 \mu \mathrm{m}$-thick aluminized Mylar, which additionally served to electrically shield the detector. The readout foil consisted of a $50 \mu \mathrm{m}$-thick Kapton substrate foil. On the charge collection side there was precisely spaced pattern of lines and pads of 0.5-1.0 oz. (18-35 $\mu \mathrm{m})$ gold-plated copper. The lines aligned vertically measured the horizontal coordinate of a hit. Between each pair of vertical lines there was a column of pads. Each pad was connected with a via to the backside of the foil where they were connected in horizontal rows to measure the vertical coordinate of a hit. The lines were $124 \mu \mathrm{m}$ wide, at a $400 \mu \mathrm{m}$ horizontal pitch. The pads were $124 \mu \mathrm{m} \times 323 \mu \mathrm{m}$, at a $400 \mu \mathrm{m}$ horizontal and vertical pitch. This geometry was chosen such that the charge collected would be approximately equally shared between the horizontal and vertical readout channels.

The signals from the lines and pads were routed to two edges of the readout foil where they terminated on sixteen arrays of pads designed to fit a flexible circuit connector, which was mounted on the front-end electronics card. Each card had four connectors (two cards per coordinate) corresponding to a total of four cards per GEM detector. Each GEM detector had 500 channels (250 per coordinate), with a total of 3000 readout channels for the GEMs in both telescopes. The front-end readout card used one APV25S1 analog pipeline chip per card 41]. Each chip had 128 channels, each of which had a 192-cell analog pipeline which sampled the input channels at $40 \mathrm{MHz}$. Data were read out of the pipeline after a trigger event. All 128 channels were multiplexed onto a single data line read out by the DAQ system. The communication between the APV card and the DAQ system was maintained by the Multi-Purpose Digitizer (MPD) 42. The MPD consisted of a VME-based module that hosted digital bus drivers, fast ADCs, and a field-programmable gate array (FPGA). The FPGA was responsible for the configuration, synchronization, triggering, and digitization of the APV cards and the data transfer along the VME bus.

The GEM detectors were fixed to an aluminum mounting bracket attached to the rails that also held the MWPCs. The mounting bracket had flexible supports for the high voltage and front-end electronics cards. These allowed the positions of the cards to be adjusted during installation to avoid interference between components. Both the mounting bracket and rails were adjustable. Survey targets located on the GEM chambers allowed the detector positions to be measured.

A charged particle traversing the GEM elements produced a charge cluster which was registered by several lines and pads in both the vertical and horizontal directions. The reconstructed centroid of the clusters in $x$ and $y$ gave the spatial location of the particle as it passed through the detector. Digitization of the signal amplitudes of all channels allowed the detector to achieve high spatial resolution $(70 \mu \mathrm{m})$. The efficiency of each GEM detector was measured with candidate tracks based on the other five telescope elements and was found to be around $95 \%$ for all GEM elements.

\subsection{2. $12^{\circ}$ Multi-Wire Proportional Chambers}

Six identical MWPC modules, along with their CROS3 readout electronics [4], were fabricated at PNPI for the $12^{\circ}$ luminosity telescopes. Each MWPC module had the external dimensions $180 \mathrm{~mm} \times 180 \mathrm{~mm} \times 50 \mathrm{~mm}$ and an active area of $112 \mathrm{~mm} \times 112 \mathrm{~mm}$. 


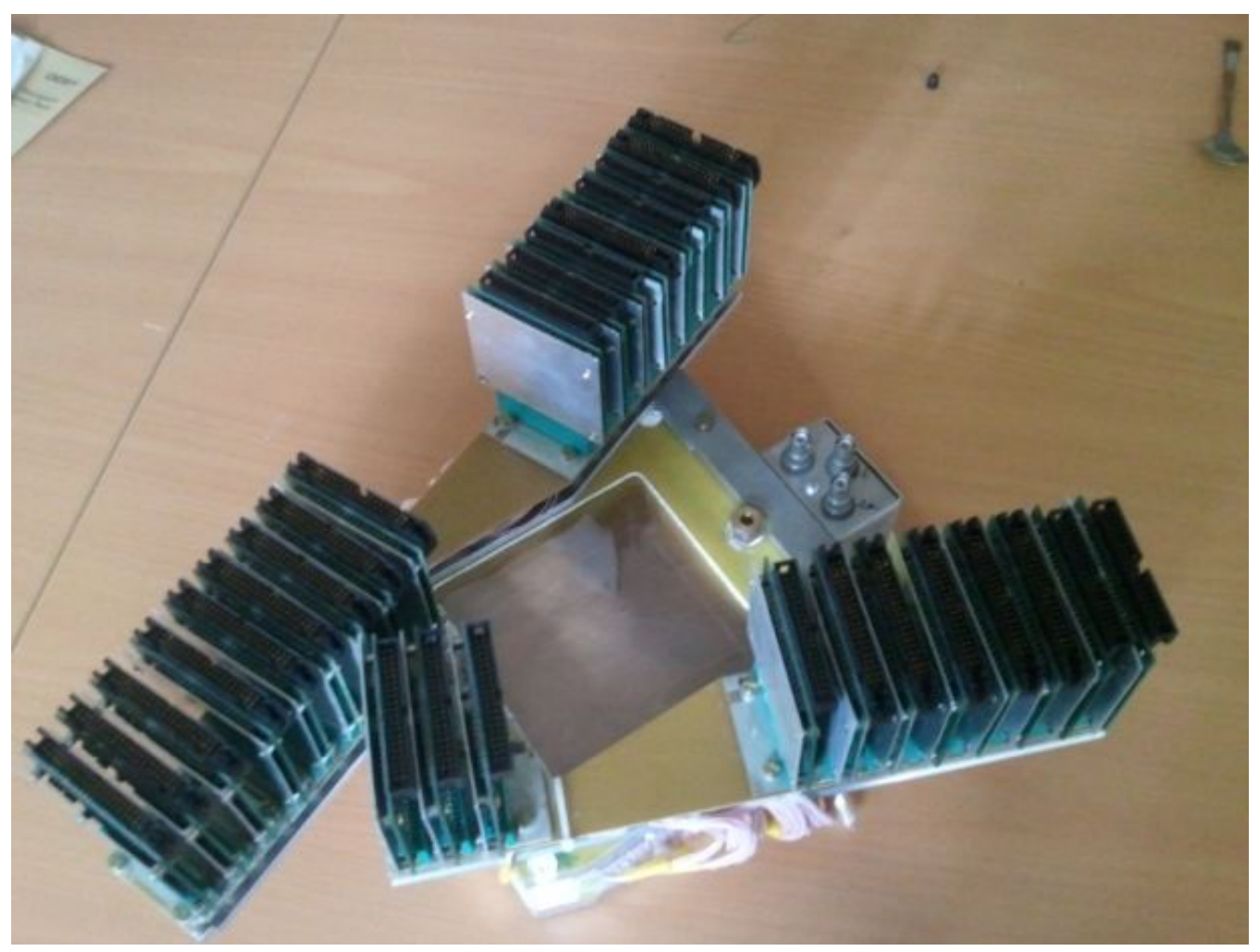

Fig. 20: Photograph of one MWPC with CROS3 readout electronics.

The readout cards for each module were arranged in two stacks around the active area, as shown in Fig. 20, to fit in the narrow space between the toroid coils.

Each MWPC module consisted of three planes of anode sense wires interleaved with cathode wire planes. The gap between the anode and cathode planes was $2.5 \mathrm{~mm}$. The anode sense wires were angled with respect to each other to allow hit reconstruction in two dimensions ( $\mathrm{X}$ vertical, $0^{\circ}, \mathrm{U}+30^{\circ}$, and $\mathrm{V}-30^{\circ}$ ). The sense wires were $25 \mu \mathrm{m}$-diameter, gold-plated tungsten separated by $1 \mathrm{~mm}$. The cathode wires were $90 \mu \mathrm{m}$-diameter beryllium bronze separated by $0.5 \mathrm{~mm}$. Each plane of wires had its own fiberglass frame. The module was assembled by sandwiching the planes together in a $10 \mathrm{~mm}$ aluminum outer frame. Each MWPC detector had material thickness of $0.25 \% \mathrm{X}_{0}$ in the active area.

A gas mixture of $\mathrm{Ar}: \mathrm{CO}_{2}: \mathrm{CF}_{4}$ (65:30:5) was chosen for the MWPCs based on the experience gained from the proportional chambers produced at PNPI for the HERMES experiment 44. GARFIELD 45] calculations predicted a gas gain of $7 \times 10^{4}$ in the MWPCs at $3150 \mathrm{~V}$. During operation, $3200 \mathrm{~V}$ was used after testing the MWPCs with a ${ }^{55} \mathrm{Fe}$ radioactive source. This operating voltage was validated during running, where an efficiency of $98-99 \%$ was typically seen for all MWPC modules. Hit distributions for each plane in a single MWPC detector are presented in Fig. 21. 


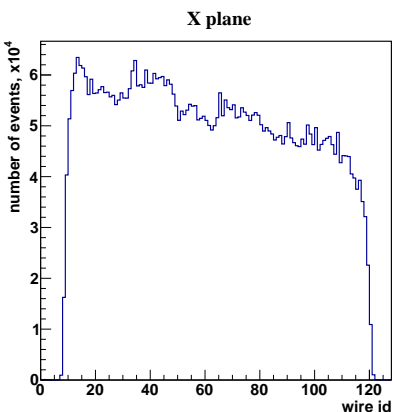

(a) X plane

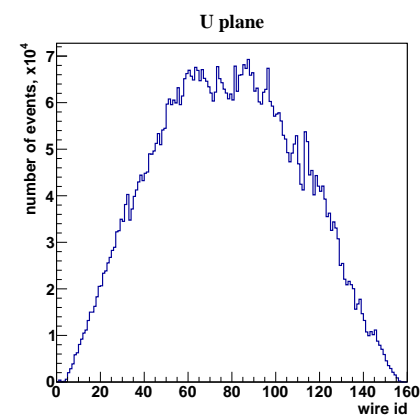

(b) U plane

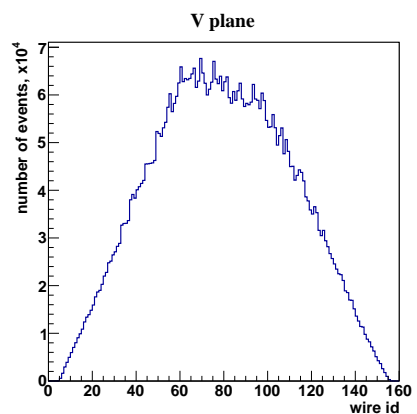

(c) V plane

Fig. 21: Hit distributions for the $\mathrm{X}, \mathrm{U}$, and $\mathrm{V}$ planes of a single, representative MWPC detector. The distribution of events is determined by the detector acceptance and sense wire angle for each plane.

\subsection{3. $12^{\circ}$ Trigger}

Each $12^{\circ}$ telescope included two $120 \mathrm{~mm} \times 120 \mathrm{~mm} \times 4 \mathrm{~mm}$ scintillator tiles (Eljen ${ }^{15}$ EJ-204) to provide a trigger signal for the GEMs and MWPCs. Each scintillator tile was wrapped in diffuse reflectors (Millipor ${ }^{16}$ Immobilon-P) and read out using two Hamamatsu ${ }^{17}$ SiPM multi-pixel photon counters (MPPCs) mounted on two opposing corners. This ensured a very high homogeneity of the light yield from the entire area of the tiles. The analog signals from each MPPC were summed and constant fraction discriminators provided the output signal from each tile. The trigger for reading out the $12^{\circ}$ telescope on a given side consisted of the triple coincidence of the two tiles on that side in conjunction with a trigger from a ToF bar in the rear region of the opposite side of the detector.

Additionally, lead glass calorimeters mounted behind the $12^{\circ}$ telescopes in each section provided an independent means of triggering the detectors. Each calorimeter consisted of three lead glass bars attached to a PMT for readout. The additional trigger contributed the ability to measure the efficiency of the tile trigger continuously throughout data taking. The two scintillator tiles in each telescope exhibited combined efficiencies in excess of $99 \%$ throughout the experimental run.

\subsection{Symmetric Møller/Bhabha Luminosity Monitor}

The symmetric Møller/Bhabha (SYMB) calorimeter measured the coincidence rate of lepton-lepton scattering events at symmetric angles. The cross sections for these processes are precisely calculable from quantum electrodynamics, and the rates in the SYMB were high enough to yield an luminosity measurement on the timescale of minutes. During electron beam running, the detector recorded Møller scattering events $\left(e^{-} e^{-} \rightarrow e^{-} e^{-}\right)$, while during positron running it was sensitive to both Bhabha scattering $\left(e^{+} e^{-} \rightarrow e^{+} e^{-}\right)$

\footnotetext{
${ }^{15}$ Eljen Technology, Sweetwater, TX, USA

${ }^{16}$ EMD Millipore, Billerica, MA, USA

${ }^{17}$ Hamamatsu Photonics K.K. Hamamatsu, Japan
} 
and annihilation $\left(e^{+} e^{-} \rightarrow \gamma \gamma\right)$ events. At the OLYMPUS beam energy of $2.01 \mathrm{GeV}$, symmetric scattering occurred at a polar angle of $1.292^{\circ}$ with respect to the beam direction (see Figs. 22 and 23 ).

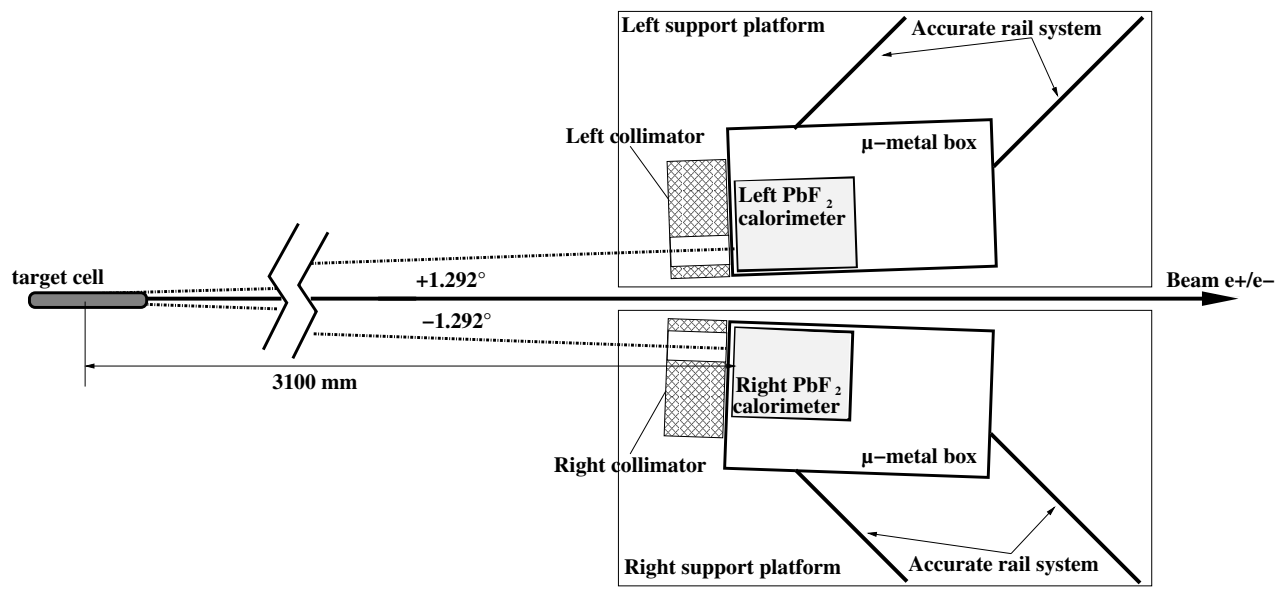

Fig. 22: A schematic of the Symmetric Møller/Bhabha luminosity detector (SYMB) showing the symmetric design about the beamline.

The SYMB, constructed at Johannes Gutenberg-Universität in Mainz, Germany, consisted of two $3 \times 3$ arrays of lead fluoride $\left(\mathrm{PbF}_{2}\right)$ crystals, as shown in Fig. 24. A Philips ${ }^{18}$ XP 29000/01 PMT was connected to the end of each crystal to provide readout. The SYMB was able to operate at high rates because of the fast response of the PMTs (20 ns), and because showers in $\mathrm{PbF}_{2}$ produce only Čerenkov radiation, eliminating the delay associated with a scintillation signal. Each crystal was approximately $26 \mathrm{~mm} \times 26 \mathrm{~mm} \times 160 \mathrm{~mm}$, with a slightly tapered shape. An array of crystals was more than 15 radiation lengths long and extended approximately 2 Molière radii from the center to the nearest edge [46]. Millipore paper wrapping around each crystal increased the surface reflectivity to reduce light loss. Each array of crystals and PMTs resided inside a mu-metal box to shield them from the magnetic fields of the OLYMPUS toroid and the DORIS beamline quadrupoles.

Lead collimators, located between each detector array and the target, shielded the crystals from beam bremsstrahlung, non-symmetric Møller/Bhabha events, and other backgrounds. Each collimator consisted of a $100 \mathrm{~mm}$ thick lead block with a precisionmachined circular hole with a diameter of $20.5 \mathrm{~mm}$. Since these apertures determined the solid angle acceptance of each detector, the location and orientation of the collimator holes was carefully surveyed before and after each running period.

The SYMB readout electronics were based on a design used for the A4 experiment at MAMI in Mainz [4]. The system provided the ability to conduct fast analog summation

${ }^{18}$ Koninklijke Philips N.V., Amsterdam, the Netherlands 


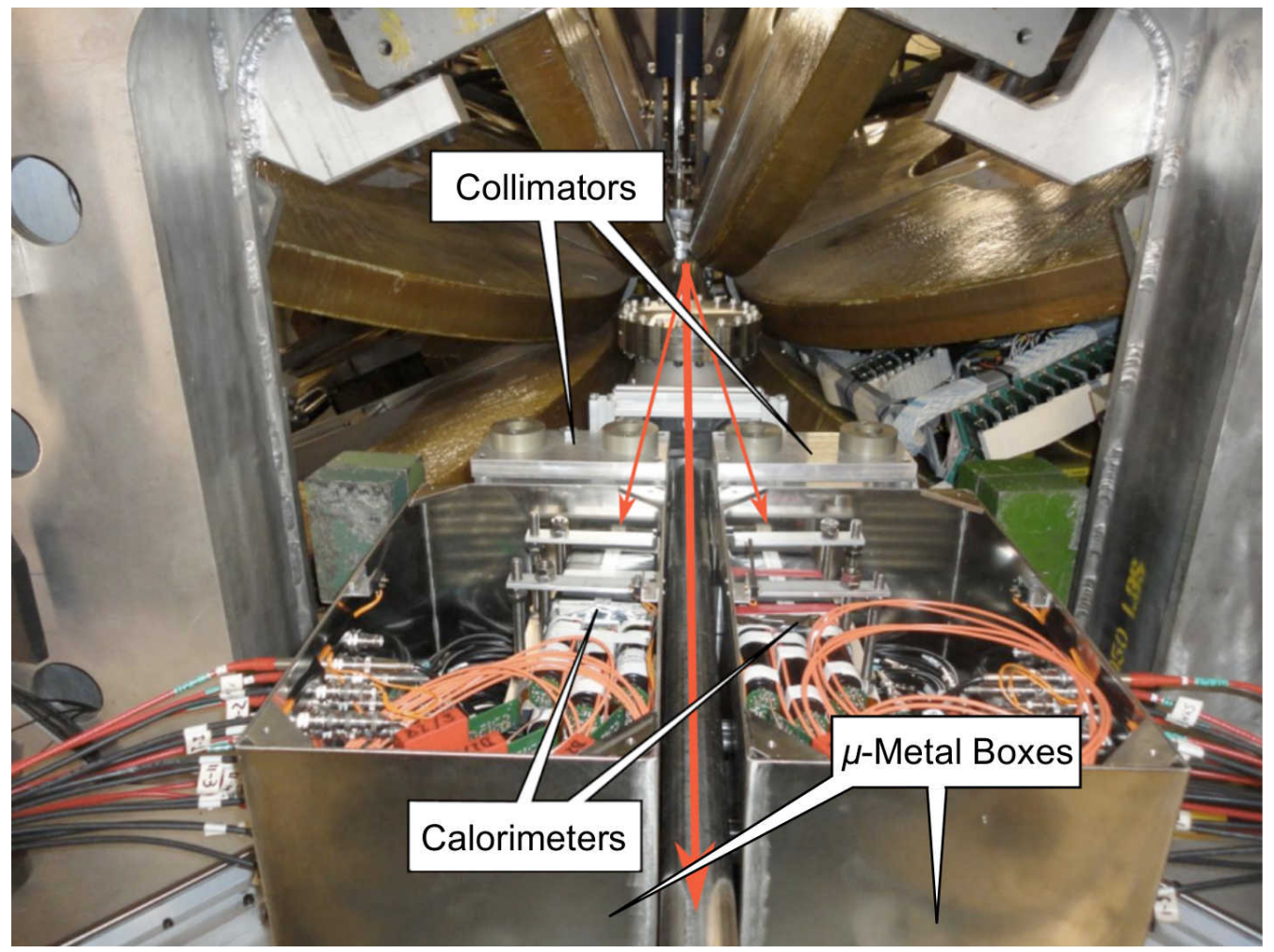

Fig. 23: A photograph showing the main components of the SYMB detector. The thick red line indicates the direction of the beam while the thinner red lines indicate the general path of scattered electrons, positrons, or photons entering the SYMB.
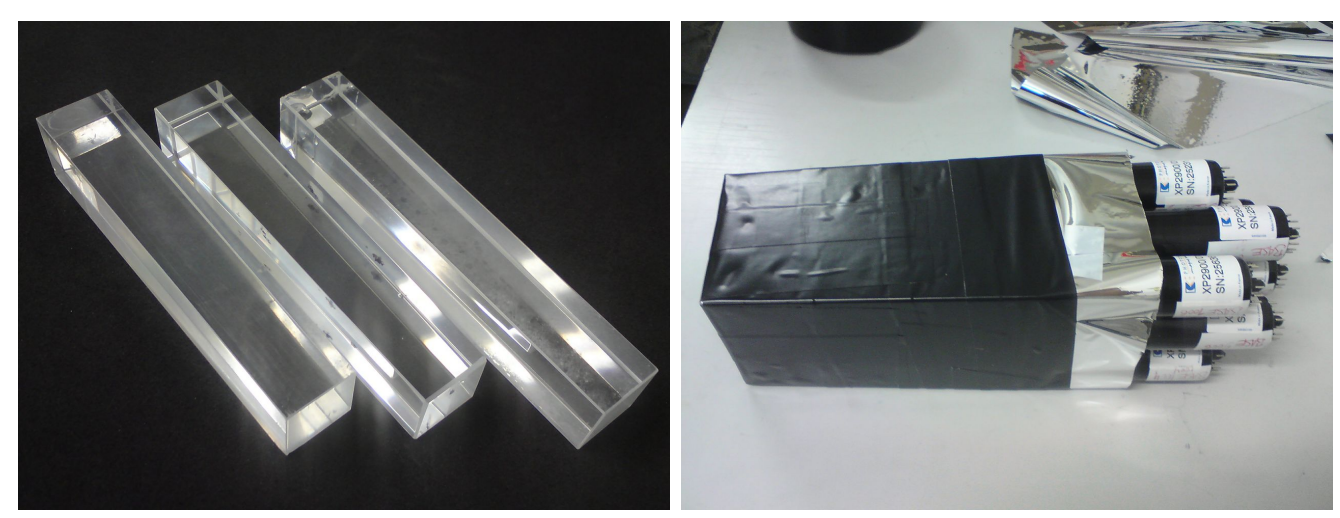

Fig. 24: Several of the $\mathrm{PbF}_{2}$ crystals used in symmetric Møller/Bhabha luminosity monitor before (left) and after (right) assembly with the PMT readout system. 
of the nine PMT signals from each crystal array and to quickly digitize and histogram the summed signal. The detector operated up to a rate of $50 \mathrm{MHz}$ (limited by the $20 \mathrm{~ns}$ signal time of the PMTs). Since the minimum bunch spacing during OLYMPUS operation was $96 \mathrm{~ns}$ there was no deadtime associated with the SYMB readout. Typical single event rates were $15 \mathrm{kHz}$, well within the operational capabilities of the readout electronics. The SYMB electronics were suspended while the OLYMPUS readout system (Sec. 6.2) processed an event to ensure the SYMB counted events only while the trigger was open.

A crystal array generated a trigger signal if two conditions were met. The first condition required that the sum of analog signals in all nine crystals exceeded the threshold of a constant fraction discriminator. The second condition required that the central crystal have the largest signal, in order to reject noise events. Upon receiving a trigger signal, the detector electronics would histogram the event. One histogram was for events when both arrays produced a trigger. Two additional histograms were filled when the left or right arrays respectively produced a trigger. Due to the high event rate, single events were not read out. Rather, the histograms were periodically sent to the data acquisition system.

Fig. 25 shows an example of the coincidence-event histogramming. Symmetric Møller,

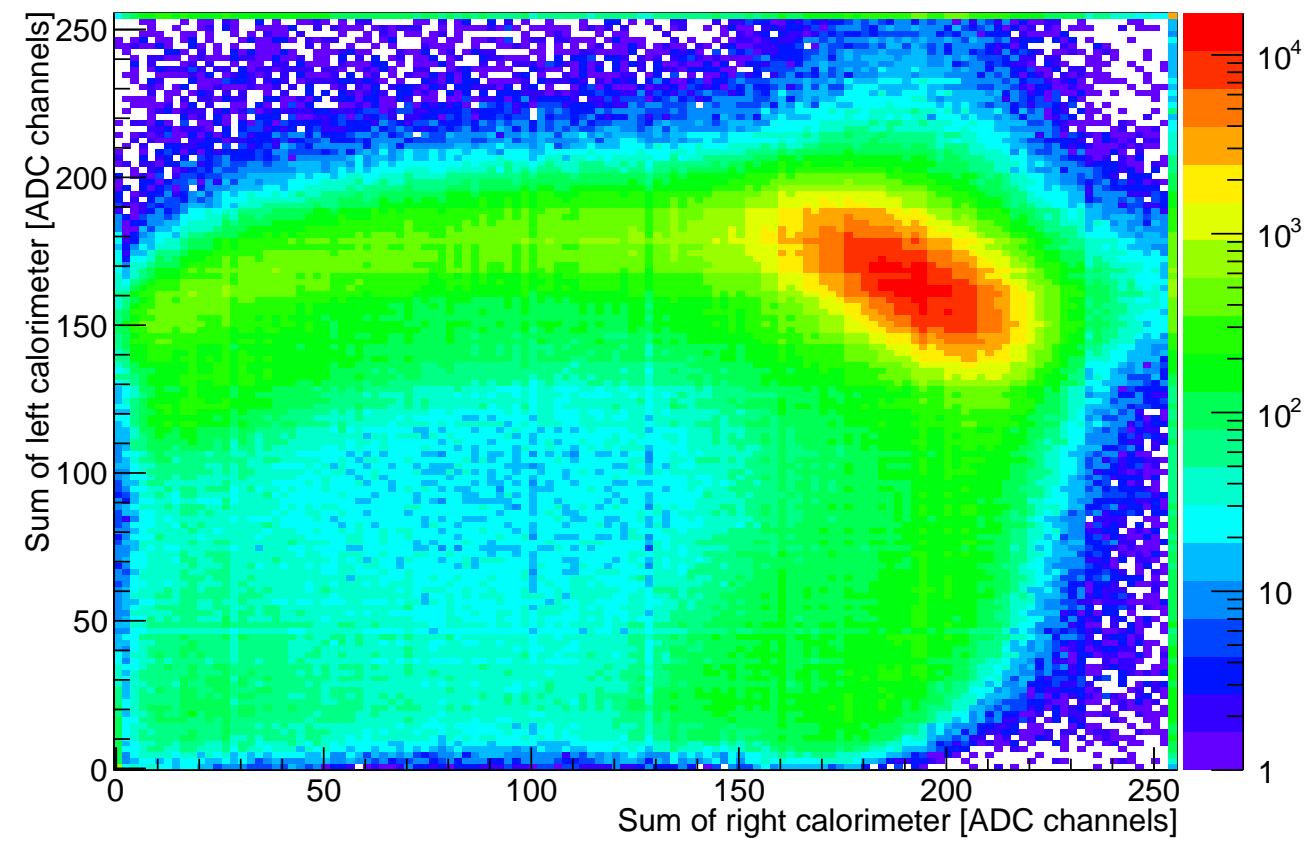

Fig. 25: A 2D histogram of the sum of the deposited energy in the left and right SYMB calorimeters in coincidence mode.

Bhabha, and annihilation events deposit approximately the same energy in both calorimeters, while many background processes deposited energy asymmetrically. 


\section{Data Acquisition}

The data readout and trigger system for OLYMPUS was developed in collaboration between the Bonn and MIT groups, based on the system originally developed for the Crystal Barrel experiment [4] at ELSA in Bonn, Germany. The system employed VME CPUs, using standard 1 GBit Ethernet for data transport, and dedicated hardware connections and modules for synchronization. Trigger logic was implemented by a flexible FPGA system. The data acquisition system was controlled through a graphical user interface. The following two subsections describe these systems in more detail.

\subsection{Trigger}

The OLYMPUS trigger system incorporated information from the time-of-flight detector, the drift chambers, the luminosity detectors, as well as information from the DORIS accelerator. This was implemented using a VME field-programmable gate array (FPGA), which combined up to 16 input signals to produce 16 parallel trigger conditions. The individual conditions could be independently prescaled.

The ToFs and the $12^{\circ}$ scintillators provided the fast trigger signals for the experiment. The primary trigger required a coincidence between a left ToF bar and a right ToF bar that could be hit by a kinematically valid elastic scattering event. Coincidence between the top and bottom PMTs in each bar was required as well. The main $12^{\circ}$ luminosity trigger consisted of a coincidence between the two $12^{\circ}$ scintillators in one sector in conjunction with a ToF in the opposite sector. The DORIS bunch clock was used to provide the reference time signal for the ToF and drift chamber TDCs.

In addition to the primary triggers, several signals corresponding to less strict ToF coincidences and signals from the lead glass calorimeters behind the $12^{\circ}$ detectors were included at higher prescale factors. Events from these triggers provided means of monitoring the efficiencies and calibration of various detector components over the course of data taking.

The data from the February run contained an unsatisfactory fraction of elastic $e^{ \pm} p$ events. A second-level trigger that incorporated information from the drift chambers was implemented for the fall run. The trigger required a signal from at least one wire in each of the middle and outer chambers on each sector and executed a fast clear of the trigger when this condition was not satisfied. This scheme reduced the false trigger rate by a factor of approximately 10 .

\subsection{Readout}

The readout system was designed and implemented by the Bonn group, based on VME CPU modules. The readout was designed in synchronous fashion. An accepted trigger would cause all detectors to be read out, while simultaneously inhibiting new triggers until the readout procedure of all detectors was completed. While a synchronous system incurs a higher deadtime than an asynchronous system, the guaranteed matching of data from different detectors for the same event and the ease of identifying readout problems outweighed this disadvantage for OLYMPUS. The detector readouts were organized in a master-slave architecture. Detectors were read out through a series of slave modules with dedicated links to a master module, which sequenced the readout. Upon receipt of a trigger, the master module would signal the slave modules to begin readout and then wait until all slave modules signaled that the procedure was completed. These signals 
were communicated over direct hardware lines while data transfer and general control were facilitated by two dedicated 1 GBit Ethernet networks.

\section{Slow Control}

The operation of the OLYMPUS experiment required several hundred parameters to be monitored, controlled, and recorded. These included high voltage supplies, vacuum pumps and gauges, the hydrogen gas supply system, the parameters of the DORIS beam, and other elements with operational time scales on the order of seconds. To satisfy these requirements, a new dedicated slow control system was developed for OLYMPUS.

The slow control system utilized the Experimental Physics and Industrial Control System (EPICS) ${ }^{19}$ as its backend solution. The system ran on three Linux machines: two VME computers with interface cards connecting to the control equipment and one server which communicated data to a PostgreSQL database and interfaced with the DORIS control system. The database recorded the status and history of all parameters associated with the slow control. The slow control also passed this information to the DAQ for integration with the detector data to produce the run data files.

The slow control system included a user-friendly, web-accessible graphical user interface, implemented using Flask ${ }^{20}$ as middleware. While typical slow control systems require the deployment of custom, operating system dependent software on their control computers, the design of the OLYMPUS system allowed both view-only and control access from any computer with an Internet connection. The user interface provided simple on-screen controls for the various elements connected to the system, displayed real-time plots and indicators of system statuses and data, and produced visual and audible alarms when parameters failed to satisfy proper run conditions.

\section{Operation}

During normal data-taking runs, a two-person shift crew operated the OLYMPUS detector and monitored the quality of the data using a number of plots generated in near real-time. Typically, production runs were taken 24 hours a day during the February and fall runs, alternating daily between positron and electrons beams. The integrated luminosity delivered to the experiment during the two runs is shown in Fig. 26 . In total, a data set of approximately $4.5 \mathrm{fb}^{-1}$ was collected over the course of both runs. As discussed in Sec. 1. the density of gas in the target cell during the February run was significantly lower than the design value because of a leak between the $\mathrm{H}_{2}$ gas feed system and the target cell. Due to this, less than $10 \%$ of the ultimate data set was collected during the February run. As is described in the following section, it was possible to run at a higher average beam current during the fall run, which allowed the experiment to reach the design goal for the integrated luminosity. At these higher currents, however, it was difficult to operate the experiment using a negative toroid polarity since low energy electrons were bent into the detectors, resulting in a very high background level. Thus, negative polarity runs were only taken occasionally, with reduced beam current and

\footnotetext{
${ }^{19}$ http://www.aps.anl.gov/epics/index.php

${ }^{20} \mathrm{http}$ //flask.pocoo.org
} 

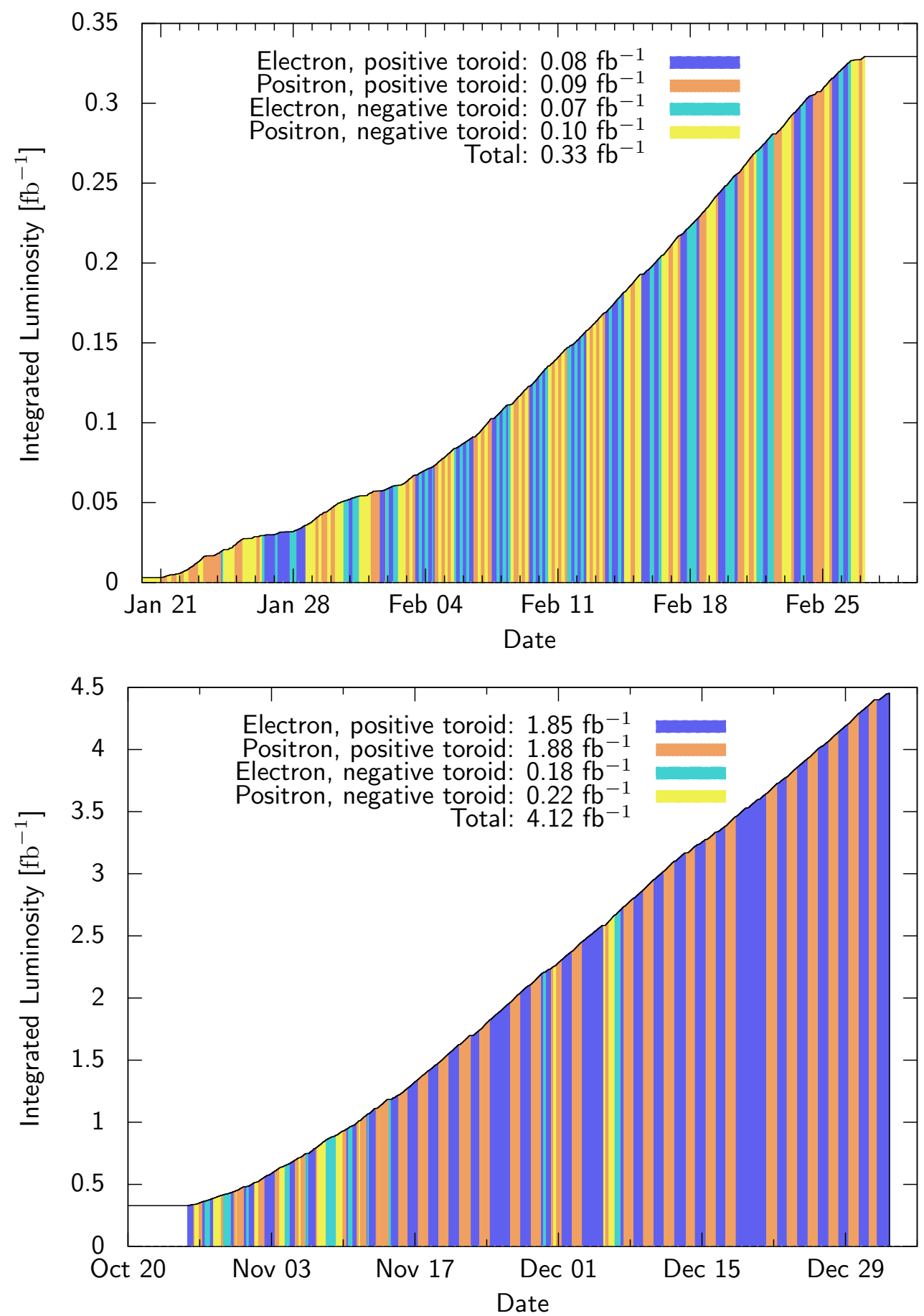

Fig. 26: The approximate integrated luminosity delivered to the OLYMPUS experiment during the February (left) and fall runs (right), as measured by the slow control (accurate to $10 \%)$. 
target flow. The uptime during the data-taking runs was extremely high (approximately $95 \%$ ), with most of the downtime due to the time required to switch the beam species (on the order of an hour).

\subsection{Data Collection}

As noted in Sec. 2, the experiment employed two modes of operation, differentiated by the manner in which the DORIS ring was operated. During the February run, the experiment was operated in "manual" mode in which the beam was initially filled to $65 \mathrm{~mA}$ and then data were taken until the beam decayed to $40 \mathrm{~mA}$. At this point, the shift crew used the slow control interface (Sec. 7) to lower the high voltage of the various detectors to safe preset values. Since beam refills during the earlier running period were not as clean as during the fall 2012 run (more instability and losses), the lowering of the voltages prevented high voltage trips and possible damage to the detectors during the refill. After lowering the voltages, the OLYMPUS shift crew informed the DORIS accelerator crew that the detector was ready for beam refill. Once the beam was restored to the normal starting current, the voltages were brought back to operational values and data-taking was restarted.

Between the February and fall runs, significant improvements were made to the DORIS beam injection process that allowed OLYMPUS to be run in "top-up mode." In this mode, the beam was initially filled to $65 \mathrm{~mA}$ as in the manual mode, but was only allowed to decay to $58 \mathrm{~mA}$ before triggering an automatic refill. Due to the improved injection, it was not necessary to lower the high voltage of the OLYMPUS detectors during these injections. The DAQ was configured to briefly inhibit data-taking during injection pulses (see Sec. 22). This mode of running significantly increased the average instantaneous luminosity delivered to the experiment and freed the OLYMPUS shift crew to more carefully monitor the quality of the incoming data.

The switch between beam species took place each morning, with occasional exceptions for maintenance and balancing the amount of data collected with each species. This ensured that there were no systematic differences between $e^{+}$and $e^{-}$runs introduced by environmental factors such as day/night cycles, reduced activity on the DESY campus on weekends, etc. During the February run, when both toroid polarities were used, datataking was segmented into four six-hour blocks each day. The pattern of toroid polarities in the four blocks each days was selected by coin toss to ensure equal running time for each polarity while avoiding systematic effects due to the time of day and week.

In addition to production runs, empty target runs (with the $\mathrm{H}_{2}$ gas flow shut-off and the target chamber pumped down to ring vacuum levels), zero magnetic field runs, and other test runs were taken on an approximately daily basis for the purposes of monitoring backgrounds, providing data for detector calibrations, and testing proposed changes to operations. When the DORIS beam was unavailable due to problems or maintenance, the detector was left active to collect cosmic ray data. Also, cosmic ray data were collected for approximately one month following the end of OLYMPUS production runs in January 2013. This large cosmic data set is being used for various studies of detector efficiencies and for calibration.

\subsection{Data Quality Monitoring}

During data taking, the quality of the incoming data was monitored in several stages. Real-time, online monitoring of essential parameters was implemented using the Ex- 
PlORA framework originally developed by the Crystal Barrel collaboration [49. The ExPIORA program processed the raw data files during data collection to produce a variety of histograms and plots of quantities versus time, such as the number of drift chamber wires hit per event, ADC and TDC distributions, DAQ deadtime, and various detector rates. The OLYMPUS shift crew had access to reference plots corresponding to those shown in ExPlORA that showed data of known good quality and data representing known possible issues. This provided the shift crew with the ability to quickly identify problems with detectors as well as problems caused by poor beam quality and take action to resolve them.

For the fall run, a second level of data quality monitoring by the shift crew was implemented that allowed inspection of the data in a more processed format approximately 30 minutes after the data was taken. This program automatically ran basic analysis programs on complete datasets as they became available and presented the data to the shift crew. In a similar fashion as the real-time monitoring, this program presented histograms and plots of the recent data to be compared with data of known quality, but included higher-level information such as the properties of events with good particle track candidates and basic measures of detector efficiencies.

Additionally, the long-term performance of the detector was monitored using the slow control database discussed in Sec. 7. This provided the ability to monitor the behavior of many detector parameters over the course of the entire data-taking period to identify slow drifts and sudden changes that could affect the analysis.

\section{Summary}

In 2012, the OLYMPUS experiment successfully collected approximately $4.5 \mathrm{fb}^{-1}$ of data for electron and positron elastic scattering from hydrogen at the DORIS storage ring at DESY. The experiment used a large acceptance, left/right symmetric detector system consisting of a toroidal magnetic spectrometer with drift chambers for tracking, time-offlight scintillators for triggering and relative timing, and a redundant set of luminosity monitors. A flexible trigger and data acquisition system was used to collect the data. The left/right symmetric design of the detector and the daily change of beam species further reduced the systematic uncertainties of the measurement. The initial plan to change the toroidal magnet polarity daily was not possible due to high background rates in the negative polarity configuration. Consequently the majority $(87 \%)$ of the data were collected with positive magnet polarity.

This paper has provided a technical description of the accelerator, internal target, detectors, data acquisition, and operation of the OLYMPUS experiment. Additional papers will detail the detector performance, analysis, and physics results.

\section{Acknowledgments}

The successful design, construction, and operation of the OLYMPUS experiment would not have been possible without the research and technical support staffs of all of the institutions involved. In particular, we would like to acknowledge the DORIS accelerator group for providing the high quality electron and positron beams delivered to the experiment. We also gratefully acknowledge the DESY MEA and MKK groups for 
providing the necessary infrastructure and support during the assembly, commissioning, operation, and disassembly of the experiment. The research and engineering group from MIT-Bates was invaluable in all phases of the experiment, from disassembling BLAST and shipping components to DESY to overcoming numerous unanticipated problems during the installation and operation of the experiment, particularly with the target and vacuum systems.

We would like to thank E. Steffens for numerous suggestions and helpful discussions during the initial development of the experiment.

Finally, we gratefully acknowledge the DESY directorate, particularly Prof. Heuer and Prof. Mnich, and the DESY Physics Research Committee for their support, advice, and encouragement from the start of the proposal.

This work was supported by numerous funding agencies which we gratefully acknowledge: the Ministry of Education and Science of Armenia, the Deutsche Forschungsgemeinschaft, the European Community-Research Infrastructure Activity, the United Kingdom Science and Technology Facilities Council and the Scottish Universities Physics Alliance, the United States Department of Energy and the National Science Foundation, and the Ministry of Education and Science of the Russian Federation. R. Milner also acknowledges the generous support of the Alexander von Humboldt Foundation, Germany.

\section{References}

[1] B. D. Milbrath, et al., Comparison of polarization observables in electron scattering from the proton and deuteron, Phys. Rev. Lett. 80 (1998) 452-455. doi:10.1103/PhysRevLett.80.452.

URL http://link.aps.org/doi/10.1103/PhysRevLett.80.452

[2] T. Pospischil, et al., Measurement of $G_{E_{n}} / G_{M_{n}}$ via polarization transfer at $Q^{2}=0.4(\mathrm{GeV} / \mathrm{c})^{2}$, Eur. Phys. J. A12 (2001) 125-127. doi:10.1007/s100500170046

[3] B. Hu, et al., Polarization transfer in the ${ }^{2} \mathrm{H}\left(\vec{e}, e^{\prime} \vec{p}\right) n$ reaction up to $Q^{2}=1.61(\mathrm{GeV} / \mathrm{c})^{2}$, Phys. Rev. C73 (2006) 064004.

[4] G. MacLachlan, et al., The ratio of proton electromagnetic form factors via recoil polarimetry at $Q^{2}=1.13(\mathrm{GeV} / \mathrm{c})^{2}$, Nucl. Phys. A764 (2006) 261-273.

[5] C. B. Crawford, et al., Measurement of the proton electric to magnetic form factor ratio from vector ${ }^{1} \overrightarrow{\mathrm{H}}\left(\vec{e}, e^{\prime} p\right)$, Phys. Rev. Lett. 98 (2007) 052301. arXiv:nucl-ex/0609007 doi:10.1103/PhysRevLett. 98.052301

[6] G. Ron, et al., Low $Q^{2}$ measurements of the proton form factor ratio $\mu_{p} G_{E} / G_{M}$, Phys. Rev. C84 (2011) 055204. arXiv:1103.5784 doi:10.1103/PhysRevC.84.055204

[7] X. Zhan, et al., High Precision Measurement of the Proton Elastic Form Factor Ratio $\mu_{p} G_{E} / G_{M}$ at low $Q^{2}$, Phys. Lett. B705 (2011) 59-64. arXiv:1102.0318 doi:10.1016/j.physletb.2011.10.002

[8] O. Gayou, et al., Measurements of the elastic electromagnetic form-factor ratio $\mu_{p} G_{E_{p}} / G_{M_{p}}$ via polarization transfer, Phys. Rev. C64 (2001) 038202. doi:10.1103/PhysRevC.64.038202.

[9] V. Punjabi, et al., Proton elastic form factor ratios to $Q^{2}=3.5 \mathrm{GeV}^{2}$ by polarization transfer, Phys. Rev. C71 (2005) 055202. arXiv:nucl-ex/0501018, doi:10.1103/PhysRevC.71.055202, 10. 1103/PhysRevC.71.069902

[10] M. K. Jones, et al., Proton $G_{E} / G_{M}$ from beam-target asymmetry, Phys. Rev. C 74 (2006) 035201 doi:10.1103/PhysRevC.74.035201 URL http://link.aps.org/doi/10.1103/PhysRevC.74.035201

[11] A. J. R. Puckett, et al., Recoil Polarization Measurements of the Proton Electromagnetic Form Factor Ratio to $Q^{2}=8.5 \mathrm{GeV}^{2}$, Phys. Rev. Lett. 104 (2010) 242301. arXiv:1005.3419, doi: 10.1103/PhysRevLett.104.242301

[12] M. Paolone, S. P. Malace, S. Strauch, I. Albayrak, J. Arrington, et al., Polarization Transfer in the ${ }^{4} \mathrm{He}\left(\vec{e}, e^{\prime} \vec{p}\right)^{3} \mathrm{H}$ Reaction at $Q^{2}=0.8$ and $1.3(\mathrm{GeV} / \mathrm{c})^{2}$, Phys. Rev. Lett. 105 (2010) 072001.

[13] A. J. R. Puckett, et al., Final analysis of proton form factor ratio data at $Q^{2}=4.0,4.8$, and 5.6 $\mathrm{GeV}^{2}$, Phys. Rev. C 85 (2012) 045203. doi:10.1103/PhysRevC.85.045203

URL http://link . aps .org/doi/10.1103/PhysRevC.85.045203 
[14] J. Litt, G. Buschhorn, D. Coward, H. Destaebler, L. Mo, R. Taylor, B. Barish, S. Loken, J. Pine, J. Friedman, G. Hartmann, H. Kendall, Measurement of the ratio of the proton form factors, $G_{E} / G_{M}$, at high momentum transfers and the question of scaling. Phys. Lett. B 31 (1) (1970) 40 - 44. doi:http://dx.doi.org/10.1016/0370-2693(70)90015-8 URL http://www.sciencedirect.com/science/article/pii/0370269370900158

[15] W. Bartel, et al., Measurement of proton and neutron electromagnetic form-factors at squared four momentum transfers up to $3(\mathrm{GeV} / \mathrm{c})^{2}$, Nucl. Phys. B58 (1973) 429-475. doi:10.1016/ 0550-3213(73) 90594-4

[16] L. Andivahis, et al., Measurements of the electric and magnetic form factors of the proton from $Q^{2}=1.75$ to $8.83(\mathrm{GeV} / \mathrm{c})^{2}$, Phys. Rev. D50 (1994) 5491-5517. doi:10.1103/PhysRevD.50.5491

[17] R. C. Walker, et al., Measurements of the proton elastic form factors for $1 \leq Q^{2} \leq 3(\mathrm{GeV} / \mathrm{c})^{2}$ at SLAC, Phys. Rev. D49 (11) (1994) 5671-5689. doi:10.1103/PhysRevD.49.5671

[18] M. E. Christy, et al., Measurements of electron-proton elastic cross sections for $0.4<Q^{2}<$ $5.5(\mathrm{GeV} / \mathrm{c})^{2}$, Phys. Rev. C70 (2004) 015206. arXiv:nucl-ex/0401030 doi:10.1103/PhysRevC. 70.015206

[19] I. A. Qattan, et al., Precision Rosenbluth measurement of the proton elastic form factors, Phys. Rev. Lett. 94 (2005) 142301. arXiv:nucl-ex/0410010 doi:10.1103/PhysRevLett.94.142301

[20] J. Bernauer, et al., The electric and magnetic form factors of the proton, submitted to Phys. Rev. C (2013). arXiv: 1307.6227

[21] P. A. Guichon, M. Vanderhaeghen, How to reconcile the Rosenbluth and the polarization transfer method in the measurement of the proton form-factors, Phys. Rev. Lett. 91 (2003) 142303. arXiv: hep-ph/0306007. doi:10.1103/PhysRevLett.91.142303

[22] P. Blunden, W. Melnitchouk, J. Tjon, Two photon exchange and elastic electron proton scattering, Phys. Rev. Lett. 91 (2003) 142304. arXiv:nucl-th/0306076, doi:10.1103/PhysRevLett.91.142304

[23] Y. C. Chen, A. Afanasev, S. J. Brodsky, C. E. Carlson, M. Vanderhaeghen, Partonic calculation of the two-photon exchange contribution to elastic electron-proton scattering at large momentum transfer, Phys. Rev. Lett. 93 (12) (2004) 122301. doi:10.1103/PhysRevLett.93.122301

[24] A. V. Afanasev, S. J. Brodsky, C. E. Carlson, Y.-C. Chen, M. Vanderhaeghen, Two-photon exchange contribution to elastic electron-nucleon scattering at large momentum transfer, Phys. Rev. D72 (1) (2005) 013008. doi:10.1103/PhysRevD.72.013008

[25] P. G. Blunden, W. Melnitchouk, J. A. Tjon, Two-photon exchange in elastic electron-nucleon scattering, Phys. Rev. C72 (3) (2005) 034612. doi:10.1103/PhysRevC.72.034612

[26] S. Kondratyuk, P. G. Blunden, W. Melnitchouk, J. A. Tjon, $\Delta$ resonance contribution to twophoton exchange in electron-proton scattering, Phys. Rev. Lett. 95 (17) (2005) 172503. doi:10. 1103/PhysRevLett.95.172503

[27] D. Borisyuk, A. Kobushkin, Box diagram in the elastic electron-proton scattering, Phys. Rev. C74 (2006) 065203. arXiv:nucl-th/0606030 doi:10.1103/PhysRevC.74.065203

[28] E. Tomasi-Gustafsson, M. Osipenko, E. Kuraev, Y. Bystritsky, Compilation and analysis of charge asymmetry measurements from electron and positron scattering on nucleon and nuclei, Phys. Atom. Nucl. 76 (2013) 937-946. arXiv:0909.4736, doi:10.1134/S106377881308022X

[29] E. Tomasi-Gustafsson, G. Gakh, Search for evidence of two photon contribution in elastic electron proton data, Phys. Rev. C72 (2005) 015209. arXiv:hep-ph/0412137 doi:10.1103/PhysRevC.72. 015209

[30] Y. M. Bystritskiy, E. A. Kuraev, E. Tomasi-Gustafsson, Structure function method applied to polarized and unpolarized electron-proton scattering: A solution of the $G_{E}(p) / G_{M}(p)$ discrepancy Phys. Rev. C75 (2007) 015207. doi:10.1103/PhysRevC.75.015207

URL http://link.aps.org/doi/10.1103/PhysRevC.75.015207

[31] M. Gorchtein, Dispersive contributions to $\mathrm{e}^{+} \mathrm{p} / \mathrm{e}^{-} \mathrm{p}$ cross section ratio in forward regime, Phys. Lett. B644 (5-6) (2007) 322-330. doi:DOI:10.1016/j.physletb.2006.11.065 URL http://www.sciencedirect.com/science/article/pii/S0370269306015231

[32] Y.-C. Chen, C.-W. Kao, S.-N. Yang, Is there model-independent evidence of the two-photonexchange effect in the electron-proton elastic scattering cross-section?, Phys. Lett. B652 (2007) 269-274. arXiv:nucl-th/0703017. doi:10.1016/j.physletb.2007.07.044

[33] J. Guttmann, N. Kivel, M. Meziane, M. Vanderhaeghen, Determination of two-photon exchange amplitudes from elastic electron-proton scattering data The European Physical Journal A - Hadrons and Nuclei 47 (2011) 1-5, 10.1140/epja/i2011-11077-4. URL http://dx.doi.org/10.1140/epja/i2011-11077-4

[34] D. Yount, J. Pine, Scattering of high-energy positrons from protons Phys. Rev. 128 (1962) 18421849. doi:10.1103/PhysRev.128.1842 
URL http://link.aps.org/doi/10.1103/PhysRev.128.1842

[35] A. Browman, F. Liu, C. Schaerf, Positron-proton scattering, Phys. Rev. 139 (1965) B1079-B1085. doi:10.1103/PhysRev.139.B1079

URL http://link.aps.org/doi/10.1103/PhysRev.139.B1079

[36] J. Mar, B. C. Barish, J. Pine, D. H. Coward, H. DeStaebler, J. Litt, A. Minten, R. E. Taylor, M. Breidenbach, Comparison of electron-proton and positron-proton elastic scattering at four-momentum transfers up to 5.0 (gev/c) ${ }^{2}$ Phys. Rev. Lett. 21 (1968) 482-484. doi:10.1103/PhysRevLett.21.482 URL http://link .aps .org/doi/10.1103/PhysRevLett.21.482

[37] B. Bouquet, D. Benaksas, B. Grossetête, B. Jean-Marie, G. Parrour, J. Poux, R. Tchapoutian, Backward scattering of positrons and electrons on protons Phys. Lett. B26 (3) (1968) $178-180$ doi:http://dx.doi.org/10.1016/0370-2693(68)90520-0

URL http://www.sciencedirect.com/science/article/pii/0370269368905200

[38] H. Albrecht, et al., Physics with ARGUS, Phys. Rept. 276 (1996) 223-405.

[39] D. Hasell, T. Akdogan, R. Alarcon, W. Bertozzi, E. Booth, et al., The BLAST experiment, Nucl. Instrum. Meth. A603 (2009) 247-262.

[40] K. A. Dow, T. Botto, A. Goodhue, D. K. Hasell, D. Loughnan, et al., Magnetic field measurements of the BLAST spectrometer, Nucl. Instrum. Meth. A599 (2009) 146-151.

[41] M. French, L. Jones, Q. Morrissey, A. Neviani, R. Turchetta, et al., Design and results from the APV25, a deep sub-micron CMOS front-end chip for the CMS tracker, Nucl. Instrum. Meth. A466 (2001) 359-365. doi:10.1016/S0168-9002(01)00589-7

[42] P. Musico, V. Bellini, M. Capogni, E. Cisbani, S. Colilli, et al., Hybrid silicon mustrip and GEM tracker for JLab hall-a high luminosity experiments, IEEE Nucl. Sci. Symp. Conf. Rec. 2011 (2011) 1306-1308. doi:10.1109/NSSMIC.2011.6154331

[43] N. Bondar, V. Golovtsov, A. Golyash, E. Lobachev, L. Uvarov, S. Uvarov, V. Yatsura, Third Generation Coordinate ReadOut System CROS-3, PNPI High Energy Physics Division Main Scientific Activities 2002-2006 (2007) 334

[44] A. Andreev, S. Belostotsky, G. Gavrilov, O. Grebenyuk, E. Ivanov, et al., Multiwire proportional chambers in the HERMES experiment, Nucl. Instrum. Meth. A465 (2001) 482-497.

[45] R. Veenhof, GARFIELD, recent developments, Nucl. Instrum. Meth. A419 (1998) 726-730.

[46] S. Baunack, et al., Realtime calibration of the A4 electromagnetic lead fluoride calorimeter, Nucl. Instrum. Meth. A640 (2011) 58-68. arXiv:nucl-ex/1102.5640 doi:10.1016/j.nima.2011.02.099

[47] R. Kothe, Design and operation of fast calorimeter electronics for an experiment for the measurement of the parity violation in elastic electron scattering, Ph.D. thesis, Johannes GutenbergUniversität, Mainz (2008).

[48] The Crystal Barrel experiment at ELSA

URL http://www1.cb.uni-bonn.de/

[49] D. M. Piontek, The new online monitor for the Crystal Barrel Experiment at ELSA, 24th Students' Workshop on Electromagnetic Interactions Bosen (Saar), 2006. 